GA-C23336

\title{
ADVANCED FUSION POWER PLANT STUDIES ANNUAL REPORT FOR 1999
}

\author{
by \\ V.S. CHAN, M.S. CHU, C.M. GREENFIELD, J.E. KINSEY, † \\ R.J. LA HAYE, L.L. LAO, Y.R. LIN-LIU, M.A. MAHDAVI, T.K. MAU,‡ \\ C.C. PETTY, P.A. POLITZER, R. PRATER, H.E. ST. JOHN, \\ M.J. SCHAFFER, G.M. STAEBLER, R.D. STAMBAUGH, \\ and A.D. TURNBULL \\ $t_{\text {Lehigh University }}$ \\ ҒUniversity of California, San Diego
}

\author{
Work supported by \\ the U.S. Department of Energy \\ under Contract No. DE-AC03-98ER54411
}

GA PROJECT 30007

JANUARY 2000 


\section{DISCLAIMER}

This report was prepared as an account of work sponsored by an agency of the United States Government. Neither the United States Government nor any agency thereof, nor any of their employees, make any warranty, express or implied, or assumes any legal liability or responsibility for the accuracy, completeness, or usefulness of any information, apparatus, product, or process disclosed, or represents that its use would not infringe privately owned rights. Reference herein to any specific commercial product, process, or service by trade name, trademark, manufacturer, or otherwise does not necessarily constitute or imply its endorsement, recommendation, or favoring by the United States Government or any agency thereof. The views and opinions of authors expressed herein do not necessarily state or reflect those of the United States Government or any agency thereof. 


\section{DISCLAIMER}

Portions of this document may be illegible in electronic image products. Images are produced from the best available original document. 


\section{TABLE OF CONTENTS}

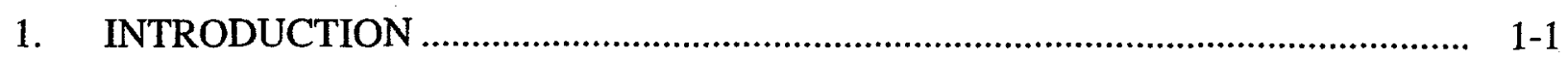

2. ARIES-RS EQUILIBRIUM AND STABILITY OPTIMIZATION ............................ 2-1

3. STABILITY TO THE RWM AND ITS STABILIZATION BY PLASMA ROTATION ............................................................................................... $3-1$

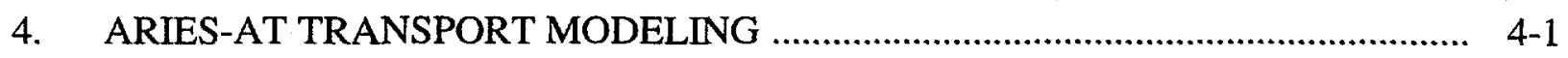

4.1. Comments on the ITB Case ........................................................................ 4-2

4.2. Future Directions ...................................................................................... 4-4

5. ECCD CONSIDERATIONS FOR ARIES-AT ……….......................................... $5-1$

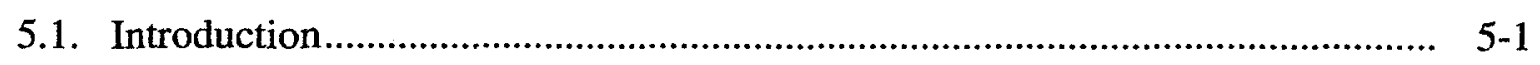

5.2. ECCD Calculations ................................................................................... $5-2$

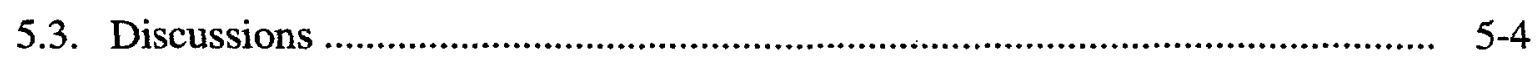

6. DIVERTOR-RELATED ISSUES FOR ARIES-AT ………...................................... 6-1

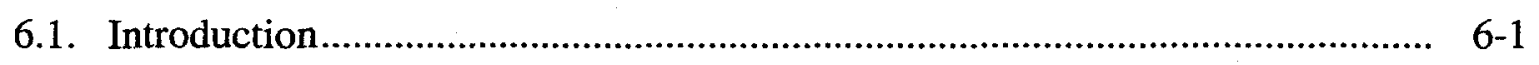

6.2. Estimation of the Peaked Heat Flux Inside the Vessel ........................................ 6-1

6.2.1. Particle heating in the divertors ............................................................... $6-1$

6.2.2. Radiated power from electromagnetic sources ......................................... 6-2

6.2.3. Application to total heat flux .............................................................. $6-2$

6.3. Additional Issues .................................................................................... 6-5

6.3.1. Magnetic balan ce of the double-null .................................................. $6-5$

6.3.2. Heat flux reduction outside the slot divertors .......................................... 6-7

6.4. Summary and Conclusions .............................................................................. 6-9 
7. CURRENT AND STORED ENERGY RAMPUP IN THE ARIES-AT TOKAMAK

7.1. Model Equilibrium....................................................................................

7.2. Rampup Scenarios .................................................................................

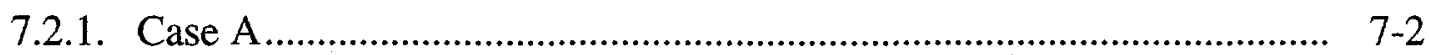

7.2.2. Case B

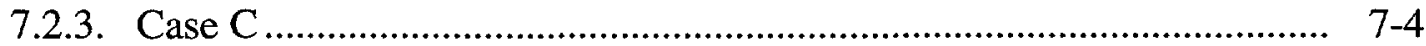

8. SUMMARY AND DISCUSSION …......................................................................

APPENDIX A 


\section{LIST OF FIGURES}

2-1. An ARIES-RS equilibrium which has a high bootstrap current fraction of $92 \%$.... 2-2

2-2. (a) Comparison of the flux-surface averaged current density and the bootstrap current density for equilibrium shown in Fig. 1 .

2-3. Variation of the normalized growth rate against the ideal $n=1$ mode with the location of a conformal conducting wall

2-4. Ballooning stability diagram for the equilibrium shown in Fig. 1

2-5. Variation of the normalized and the toroidal betas with the shaping parameter $\mathrm{S}=\mathrm{I}_{\mathrm{N}} \mathrm{q} 95$.

2-6. An ARIES-RS equilibrium with a lower current and a lower stored energy which has a high bootstrap current fraction of $96 \%$

2-7. Comparison of the flux-surface averaged current density and the bootstrap current density for the equilibrium shown in Fig. 6

2-8. Ballooning stability diagram for the equilibrium shown in Fig. 6 ........................ $2-6$

3-1. Amplitudes of the harmonics of the perturbed magnetic field $\delta B_{\psi}$ as a function of $\sqrt{V}$ for equilibrium g099728.00270.

3-2. Stability window in $r_{w}$ for the resistive wall mode for equilibrium g099728.00270

4-1. Note that $T_{i}=T_{e}$ for the base case

4-2. $I_{\text {boot }} / I_{p}=81 \%$ for the base case and $I_{\text {boot }} / I_{p}=96 \%$ for the ITB case

5-1. Calculated on-axis current drive with EC waves in typical ARIES-AT equilibrium (a) ray trajectories, and (b) driven current profile

5-2. Calculated off-axis current drive with EC waves in typical ARIES-AT equilibrium (a) ray trajectories, and (b) driven current profile

6-1. The peak heat flux delivered to each DIII-D divertor is a sensitive function of drSEP.

6-2. (a) The $1.5 \mathrm{~cm}$ flux surface (as measured from the outboard midplane) intersects the baffle roof.

A-1. Three helicity injection concepts. 


\section{LIST OF TABLES}

4-1. Summary of transport results ....................................................................

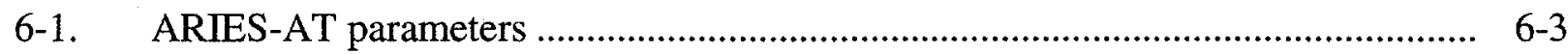




\section{INTRODUCTION}

Significant progress in physics understanding of the reversed shear advanced tokamak regime has been made since the last ARIES-RS study was completed in 1996. The 1999 study aimed at updating the physics design of ARIES-RS, which has been renamed ARIES-AT, using the improved understanding achieved in the last few years. The new study focused on

- Improvement of beta-limit stability calculations to include important non-ideal effects such as resistive wall modes and neo-classical tearing modes

- Use of physics based transport model for internal transport barrier (ITB) formation and sustainment

- Comparison of current drive and rotational flow drive using fast wave, electron cyclotron wave and neutral particle beam

- Improvement in heat and particle control

- Integrated modeling of the optimized scenario with self-consistent current and transport profiles to study the robustness of the bootstrap alignment, ITB sustainment, and stable path to high beta and high bootstrap fraction operation.

In the following sections, we present important results in key topical areas. 


\section{ARIES-RS EQUILIBRIUM AND STABILITY OPTIMIZATION}

The equilibria are computed using the previous 1996 ARIES-RS reference equilibrium [2-1] as a starting point and the MHD equilibrium codes EFIT and TOQ. The poloidal coil set, and the pressure profile

$$
P(\psi)=\alpha_{0}\left(1-\psi_{\mathrm{n}} \gamma_{\text {in }}\right)^{\gamma_{\text {out }}}
$$

are similar to those in the 1994 design. The poloidal current profile $\mathrm{F}(\psi)$ is modeled differently and is chosen to give a self-consistent bootstrap current profile over most of the plasma volume based on the approximate bootstrap model as described in [2-2]

$$
\langle\mathrm{J} \bullet \mathrm{B}\rangle_{\text {bootstrap }}=\mu_{0} \mathrm{~g}\left(\psi_{\mathrm{n}}\right) \mathrm{FP}^{\prime}(\psi)
$$

A fixed boundary equilibrium is first computed using TOQ. EFIT is then used to compute a free boundary equilibrium as closely matched to the TOQ results as possible with the given external poloidal coil set. Ideal low $n=1-5$ and high $n$ ballooning stability are then evaluated using the GATO and the BALOO codes.

A free boundary equilibrium computed this way is shown in Fig. 2-1. This particular equilibrium has a $\beta_{\mathrm{N}}=5.57$ and $\beta_{\mathrm{T}}=6.7 \%$. The plasma current and vacuum toroidal magnetic field are $\mathrm{I}_{\mathrm{P}}=13.2 \mathrm{MA}$ and $7.98 \mathrm{~T}$ at the plasma center. The equilibrium is constrained to have a high bootstrap current fraction of $92 \%, \gamma=\mathrm{L}_{\mathrm{P}} / \mathrm{L}_{\mathrm{T}}$ is $0.6, \mathrm{Z}_{\mathrm{eff}}=1.69$, $\gamma_{\text {in }}=1.7, \gamma_{\text {out }}=2.5$. This is illustrated in Figs. 2-2(a) and 2-2(b).

Ideal low $n=1-5$ modes are stabilized by a conducting wall. This is illustrated in Fig. 2-3, where the normalized growth rates against the $n=1$ mode with a conformal conducting wall at various distances away from the plasma are shown. As shown in the figure, the $n=1$ mode becomes stable with a conducting wall closer than $1.4 \mathrm{a}$. The $n=2-5$ modes are stable with a conducting wall at $1.2 \mathrm{a}$.

The MHD stability of this equilibrium is limited by ballooning modes in the plasma outer region. This is illustrated in Fig. 2-4, where the normalized pressure gradient $\alpha$ is compared against the ideal ballooning stability boundary. As shown in the figure, this configuration has second ballooning stability access over most of the plasma inner volume, except in the edge where it is marginally stable to the ballooning mode.

The toroidal beta $\beta_{\mathrm{T}}$ increases with the plasma shaping but the normalized beta $\beta_{\mathrm{N}}$ varies only weakly. This is illustrated in Fig. 2-5, where the variation of $\beta_{\mathrm{N}}$ and $\beta_{\mathrm{T}}$ are 
shown as a function of the shaping parameter $S=\mathrm{I}_{\mathrm{N}} 95$. Also shown in Fig. 2-5 are the results from a previous DIII-D NCS study [2-3].

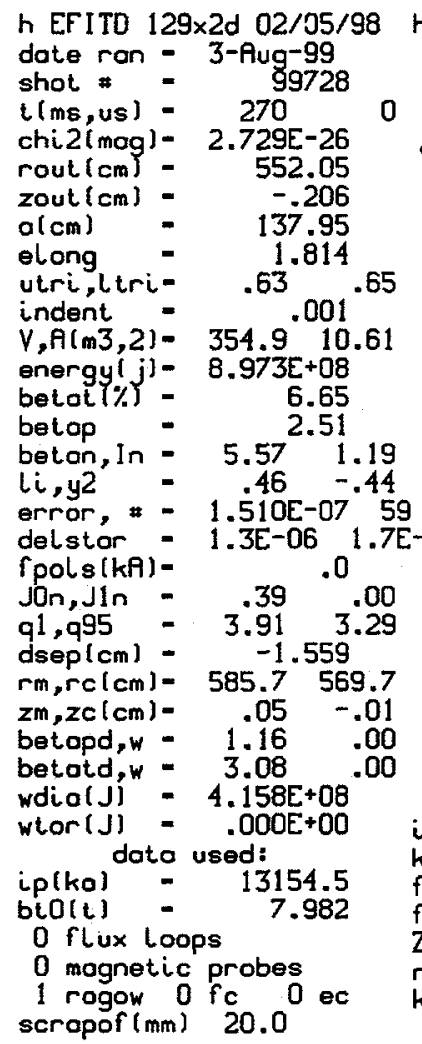

h $\quad$ 099728.00270?55

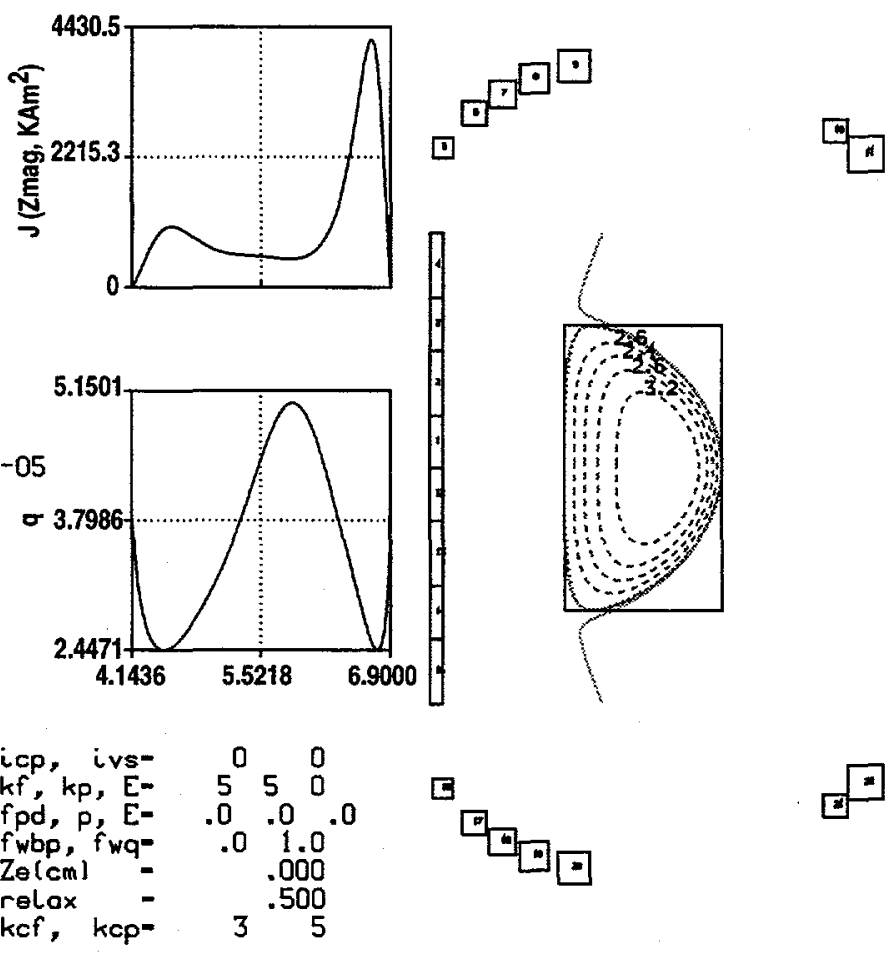

Fig. 2-1. An ARIES-RS equilibrium which has a high bootstrap current fraction of $92 \%$.

The equilibrium shown in Fig. 2-1 has a stored energy of $897 \mathrm{MJ}$ and a $\beta_{\mathrm{T}}=6.7 \%$. With high bootstrap fraction the plasma stored energy and the plasma current are strongly coupled. To reduce the stored energy it is necessary to reduce IP. An equilibrium with a lower stored energy of $500 \mathrm{MJ}$ and a lower $\mathrm{I}_{P}=8.3 \mathrm{MA}$ is shown in Fig. 2-6. The equilibrium has a high bootstrap current fraction of $96 \%, \gamma=\mathrm{L}_{\mathrm{P}} / \mathrm{L}_{\mathrm{T}}$ is $0.8, \mathrm{Z}_{\mathrm{eff}}=1.69$, $\gamma_{\text {in }}=1.7, \gamma_{\text {out }}=2.5$. This is illustrated in Fig. 2-7. For this case no edge current drive is required. Again, the MHD stability is limited by ballooning modes in the plasma outer region. This is shown in Fig. 2-8. Note that compared with the higher current case shown in Fig. 2-1, q95 is increased from 3.3 to 5.6. $\mathrm{q}_{\mathrm{min}}$ is also increased from 2.45 to 3.52 which should improve stability against the neo-classical tearing modes. 

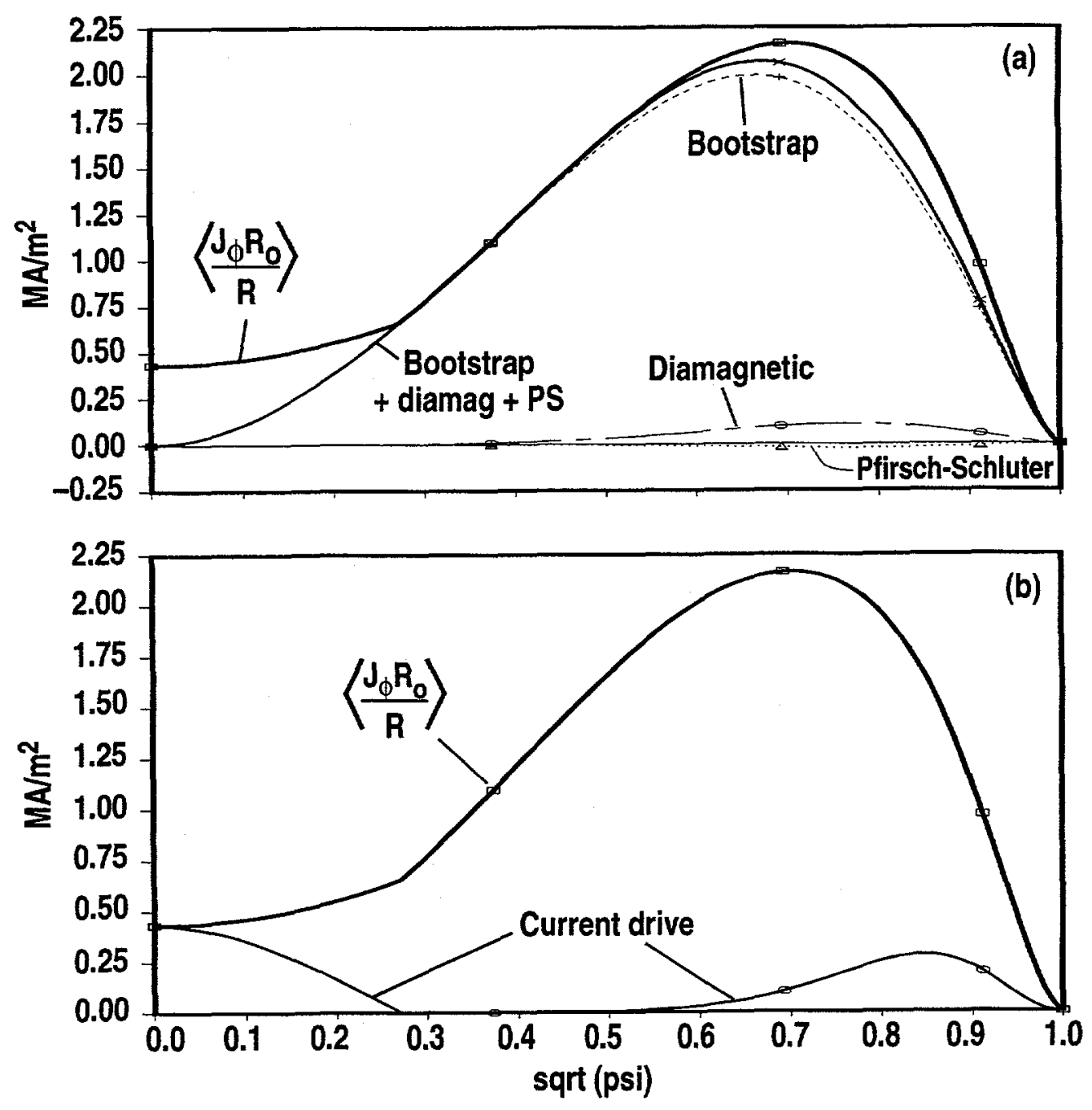

Fig. 2-2. (a) Comparison of the flux-surface averaged current density and the bootstrap current density for the equilibrium shown in Fig. 1. The bootstrap current fraction is $92 \%$. (b.) Comparison of the flux-surface averaged current density and the current density which needs to be driven externally. 


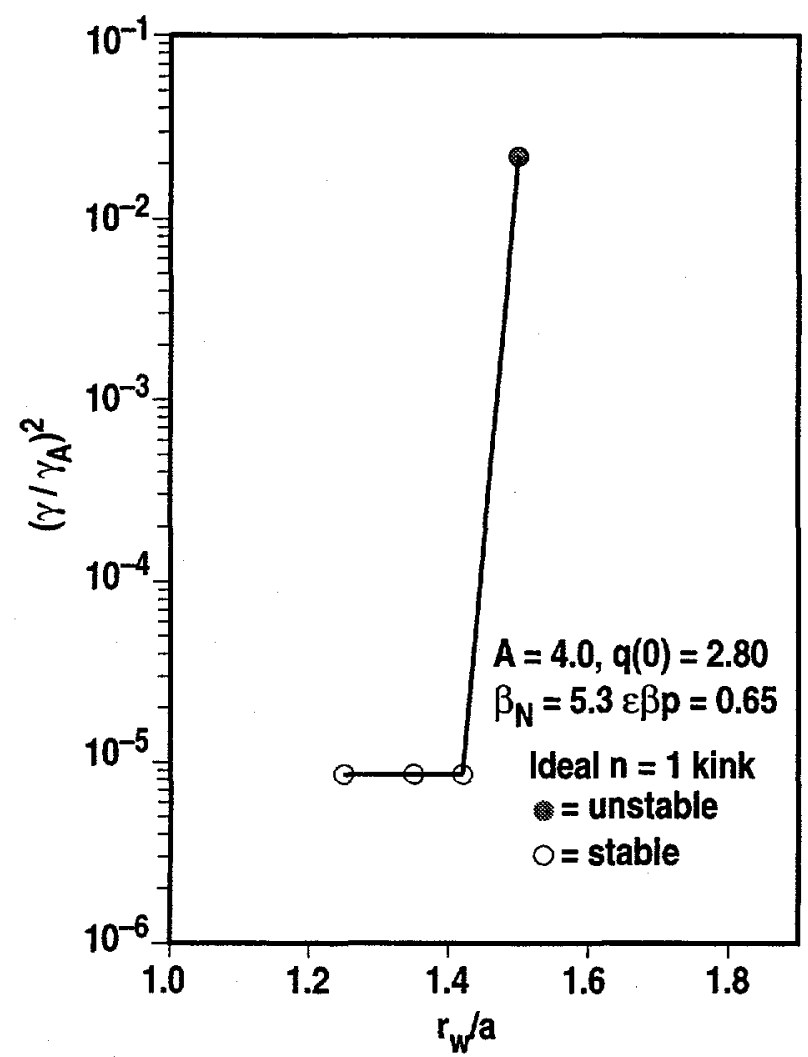

Fig. 2-3. Variation of the normalized growth rate against the ideal $n=1$ mode with the location of a conformal conducting wall.
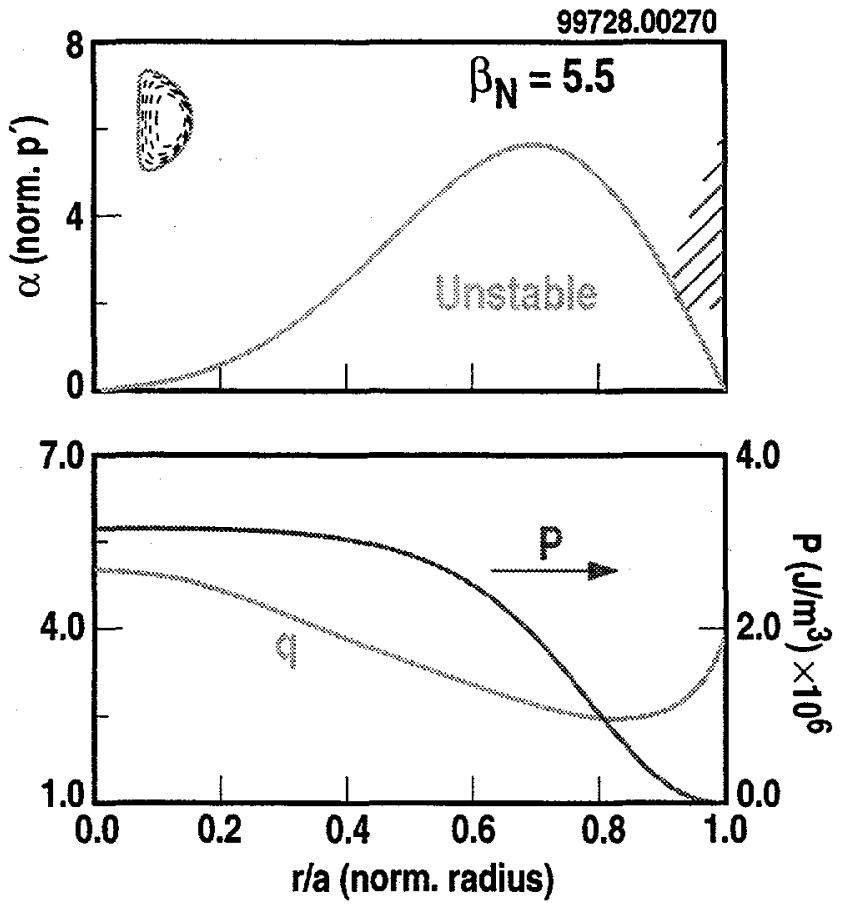

Fig. 2-4. Ballooning stability diagram for the equilibrium shown in Fig.1. Also shown are the pressure and the q profiles. 

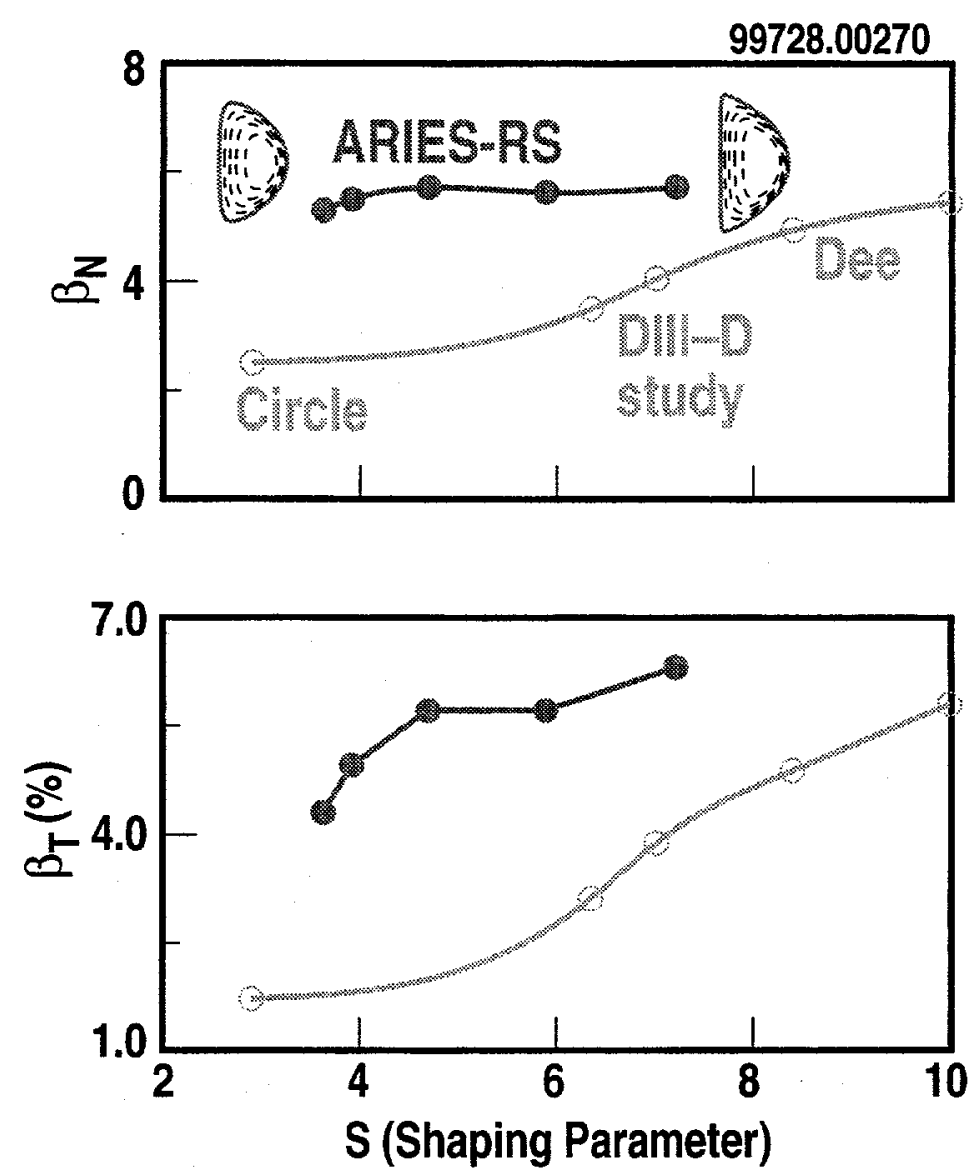

Fig. 2-5 Variation of the normalized and the toroidal betas with the shaping parameter $S=I_{N} 995$.

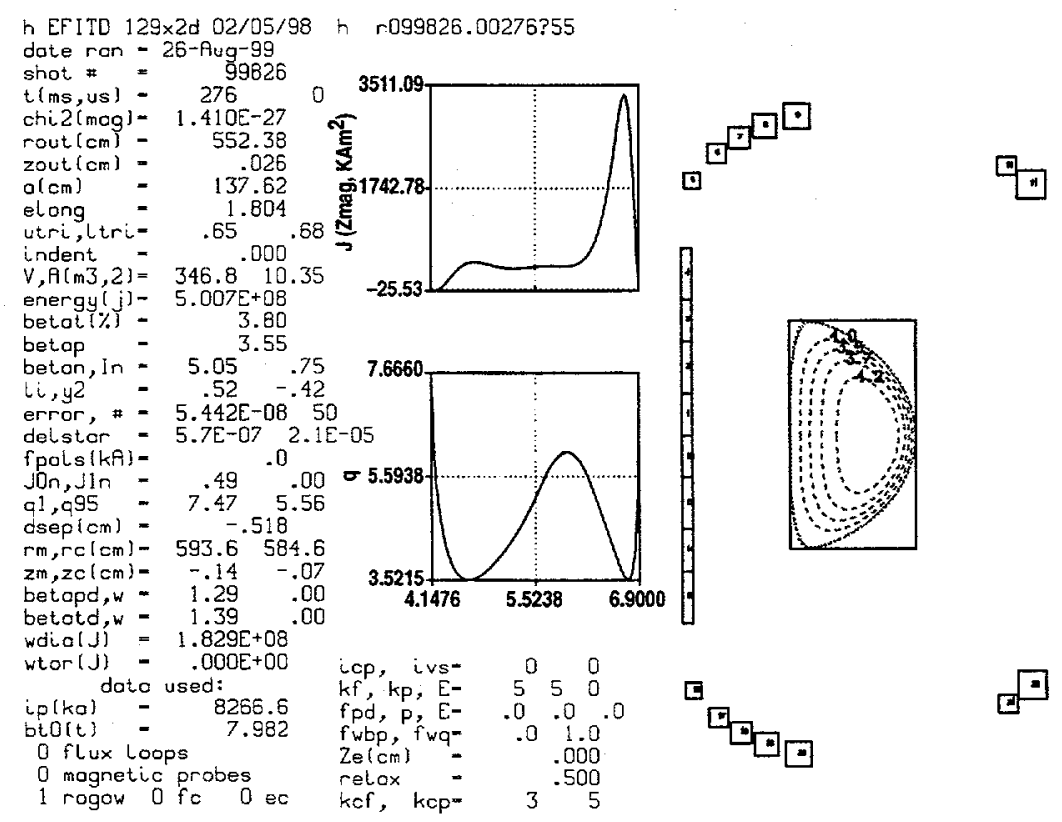

Fig. 2-6. An ARIES-RS equilibrium with a lower current and a lower stored energy which has a high bootstrap current fraction of $96 \%$. 

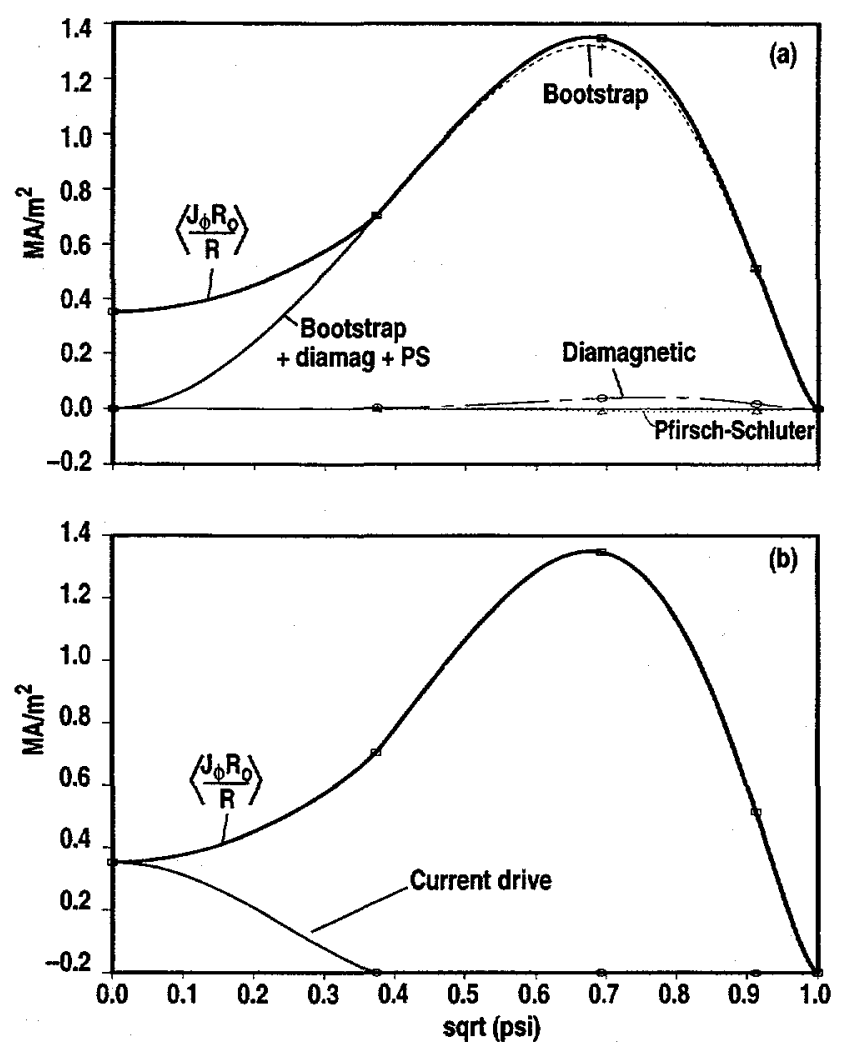

Fig. 2-7. Comparison of the flux-surface averaged current density and the bootstrap current density for the equilibrium shown in Fig. 6. The bootstrap current fraction is $96 \%$. Also shown is the current density which needs to be driven externally.
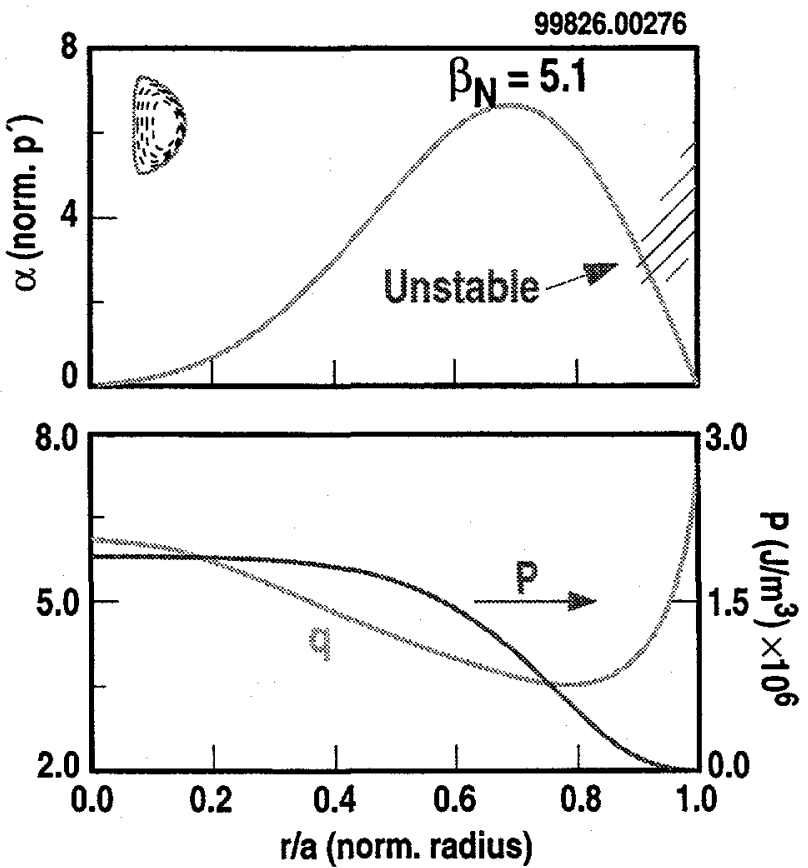

Fig. 2-8. Ballooning stability diagram for the equilibrium shown in Fig.6. Also shown are the pressure and the $q$ profiles. 


\section{REFERENCES}

[2-1] S.C. Jardin, et al, Fusion Engineering and Design 38 (1997) 27.

[2-2] R.L. Miller, et al, Phys. Plasmas 4 (1997) 1062.

[2-3] A.D. Turnbull, et al, Nucl. Fusion 38 (1998) 1467. 


\section{STABILITY TO THE RWM AND ITS STABILIZATION BY PLASMA ROTATION}

Two proposed ARIES-ST equilibria, g099728.00270 or Case (1) and g099326.00600 or Case (2), were tested for stability to the RWM and stabilization by plasma rotation by using the MARS stability code. Because of (1) there is a lack of information on the expected rotation profile, (2) for reasons of simplicity and (3) recently there was research activity on the possible utilization of a rotating liquid wall, we assume that the plasma has a constant rotation velocity across its cross-section. The model for the damping of the toroidal momentum used is the sound wave damping model. In this model, there is a force which damps the perturbed toroidal motion of the mode according to the formula

$$
\mathrm{F}_{\mathrm{SD}}=-\mathrm{k}_{\|} \sqrt{\pi}\left|\mathrm{k}_{\|\|} v_{\mathrm{th}_{\mathrm{i}}}\right| \rho \overrightarrow{\mathrm{v}} \bullet \hat{\mathrm{b}} \hat{\mathrm{b}}
$$

Here, $k_{\|}$is a numerical coefficient with a value of 1.77 to model the ion Landau damping process, $k_{\| l}$ is the parallel wave number $(m-n q) / R, v_{t h_{i}}$ is the ion thermal velocity, $\rho$ is the mass density, $\vec{v}$ is the perturbed plasma velocity and $\hat{b}$ is the unit vector of the equilibrium magnetic field.

Main results of the investigation indicate that the critical rotation velocity required for the stabilization of the resistive wall mode in Case (2) is much higher than that for Case (1). This critical rotation velocity for Case (1) is 0.065 of the Alfvén velocity, whereas it is around 0.3 times of the Alfvén velocity for Case (2). The reason for this has been traced to the presence of more rational surfaces in Case (1) than in Case (2). This is because, according to the sound wave damping model, the presence of a higher pressure at the rational surfaces will induce higher damping to the sound wave at the side band resonance locations. This will in turn induce phase-shift to the wave and unlock the mode from the resistive wall.

Case (1) has four resonant surfaces for $n=1$, at $\tilde{\psi}=0.64 \times 10^{-3}, 0.07,0.25$ and 0.89 . Aside from the first resonant surface which is located at the plasma center (where we expect the unstable mode to have very small mode amplitude), we expect all resonant surfaces to contribute.

Case (2) has only one resonant surface for $n=1$, at $\tilde{\psi}=0.86$.

Shown in Fig. 3-1 is the mode amplitude of the various harmonics of the resistive wall mode as a function of the square root of the plasma volume. It is observed that this mode has quite a global mode structure. A plot of the growth rate of the resistive wall mode as a function of the location of the resistive wall with the relative rotation 
frequency of the plasma with respect to the resistive wall as a parameter for Case (1) is shown in Fig. 3-2. To further reduce the value of the critical rotation velocity, it is expected that an $\mathrm{H}$-mode edge with multiple resonant surfaces at the edge will help.

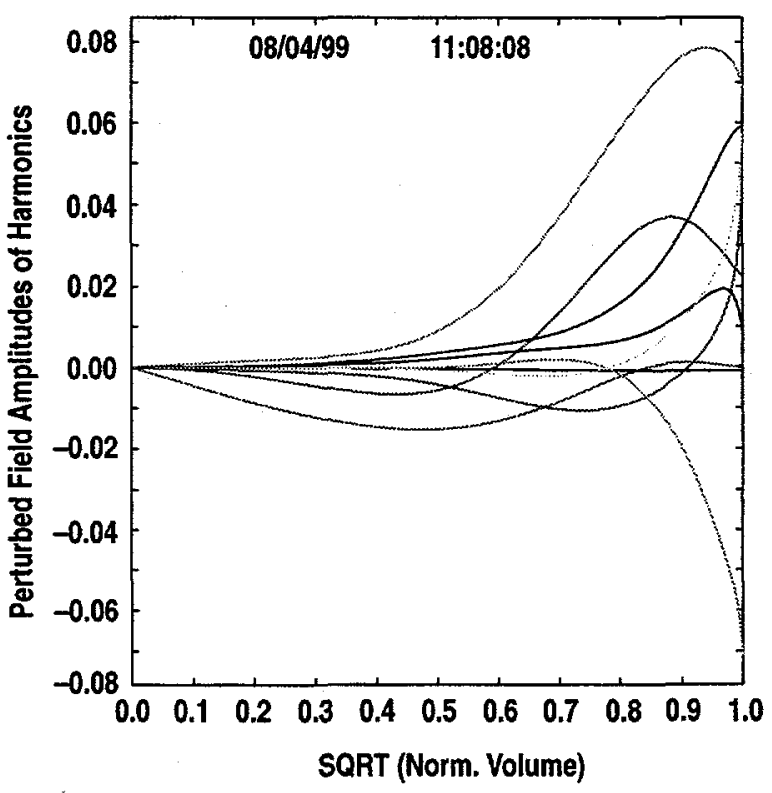

Fig. 3-1. Amplitudes of the harmonics of the perturbed magnetic field $\delta B \psi$ as a function of $\sqrt{V}$ for equilibrium g099728.00270, Case (1) for a uniformly rotating plasma with $\Omega=0.068$ measured in Alfuén frequency and $r_{w}=1.2 r_{p}$.

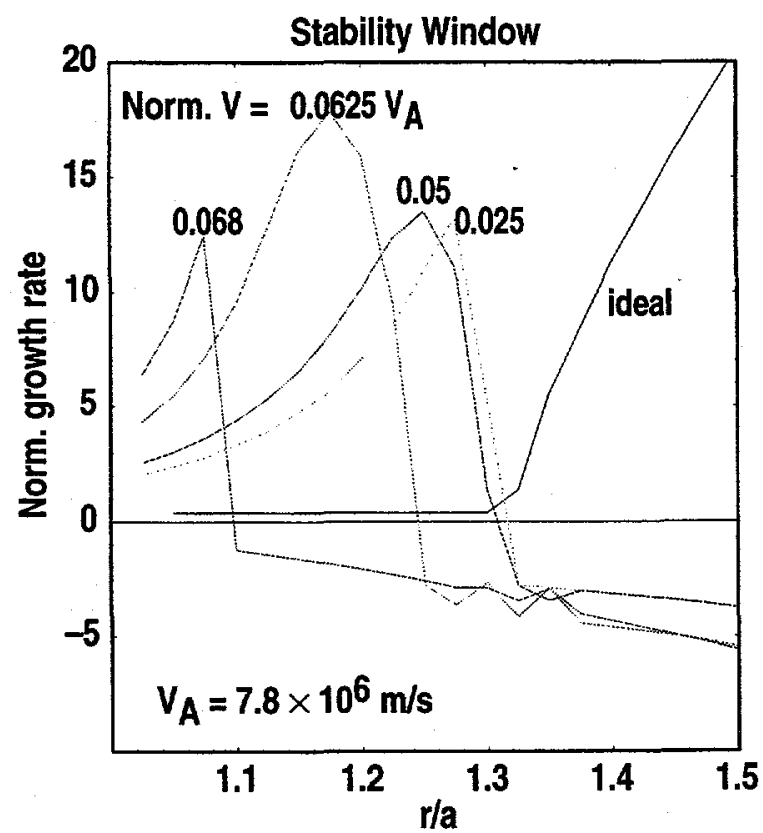

Fig. 3-2. Stability window in $r_{w}$ for the resistive wall mode for equilibrium g099728.00270, Case (1). Plotted are growth rates of the ideal and resistive wall modes versus $r_{w}$. The curve labelled ideal the growth rate of the ideal mode with the scale multiplied by 200. The other curves are labelled by the fraction of the rotation velocity versus the central Alfvern velocity. The growth rate of the resistive wall modes are multiplied by $\tau_{w}$. 


\section{ARIES-AT TRANSPORT MODELING}

Transport modeling of the ARIES-RS plasma used a multi-code strategy with the following steps:

1. A bootstrap aligned MHD equilibrium is found using the TOQ code.

2. The ONETWO transport code is run to compute the fusion power and any auxiliary power source for the equilibrium density and temperature profiles.

3. The GLF23 transport model is used (in a separate transport code) to find the steady state temperature profiles holding the density and power sources fixed. The fusion power is reduced if needed to keep the pressure near the target beta.

4. The GLF23 temperature profiles are transferred to ONETWO and the fusion power is recomputed. The deuterium/tritium ratio is adjusted in order to match the fusion power reduction required to match beta.

5. Iteration between 3 and 4 proceeds until convergence is achieved.

It was necessary to run the GLF23 model in a separate transport code because the model requires specialized numerical methods, which have not yet been implemented into the ONETWO code. The GLF23 transport model [4-1] is a state-of-the-art drift wave transport model. The model is uniquely suited for use in this study as it is able to include both the $\mathrm{E} \times \mathrm{B}$ shear suppression of ion thermal transport (transport barrier) and the anomalous electron thermal transport which remains within the transport barrier.

The GLF23 transport model uses the linear instabilities of the gyro-Landau fluid equations. These equations approximate the full gyro-kinetic theory. The transport fluxes are computed from quasilinear theory with a mixing length model for the saturated fluctuation level. The fitting parameters of the model are all fit to kinetic linear theory and to non-linear simulations of ion temperature gradient (ITG) and trapped electron mode (TEM) turbulence. No fitting to experiment has been done. Ten wavenumbers are used for the ITG-TEM modes and 10 for the electron temperature gradient (ETG) modes at high wavenumbers. The GLF23 model reproduces the L-mode and $\mathrm{H}$-mode profiles from the ITER database to within about $20 \%-30 \%$. This primarily tests the ITG-TEM transport. The GLF23 model has not been compared to a large database of internal barrier discharges, so the ETG mode, which determines the electron transport within the internal transport barrier (ITB), has not been extensively tested. It has been shown to be 
reasonable for a limited number of DIII-D discharges. The threshold level of ExB velocity shear needed to quench the ITG-TEM modes is taken from theory but has not been extensively tested against data with the GLF23 model.

The parameters from four cases are summarized in Table 4-1. These four cases represent the evolution in the design point which was driven by the transport code results. The first case in Table 4-1 is the 13.2 MA original high beta TOQ equilibrium from the MHD stability study. This case has very good bootstrap alignment and a high stability limit. However, it was found that the alpha heating power produced was much too high and that the density was 2.29 times the empirical Greenwald density limit ( $\mathrm{n}_{\mathrm{eGW}}$ ). The energy confinement for this case is also quite low. Just lowering the density to the Greenwald limit (preserving the total pressure profile of the equilibrium) gives the second column in Table 4-1. This case has too low of a bootstrap fraction (61\%) because the bootstrap current decreased with the decrease in $\mathrm{n} / \mathrm{T}$ at fixed pressure. The fusion power is also still high. These first two cases are also very close to the MHD stability limit, which is not the reactor operating point, so beta should be lowered. This would further reduce the bootstrap current. In order to get high bootstrap fraction at lower density and beta, a lower current equilibrium was found. The choice of the current was made by scaling the 13.2 MA case in ONETWO to lower beta and lower density. The current was then lowered until the bootstrap fraction was raised to $80 \%$. A new TOQ equilibrium was then calculated at this current. The results from ONETWO run with this new base equilibrium are shown in the third column of Table 4-1. The fusion power and beta for this case are more reasonable. The density is still 1.4 times the Greenwald limit. It was found that lowering the density to the Greenwald limit required dropping the current unacceptably low to regain $80 \%$ bootstrap fraction. This is because the Greenwald limit scales linearly with current. Starting from this new base case, the GLF23 transport model was run with an $\mathrm{E} \times \mathrm{B}$ shear induced internal transport barrier (ITB) inside of $\mathrm{r} / \mathrm{a}=0.8$. Inside the ITB the ion thermal transport is neoclassical but the electron thermal transport is due to ETG modes. The energy confinement improved compared to the base case, making it necessary to raise the $\mathrm{D} / \mathrm{T}$ (deuterium/tritium) ratio to $90 \%$ in order to lower the fusion power and keep the beta constant.

\subsection{COMMENTS ON THE ITB CASE}

The temperature and density profiles for the 8.27 MA base case and the ITB case are shown in Fig. 4-1. The large Shafranov shift and negative magnetic shear in the center of the plasma is stabilizing the ETG mode. This causes the electron temperature profile to 
peak near the axis. This results in poor bootstrap alignment as shown in Fig. 4-2. In NCS experiments with ITB's on DIII-D, the electron temperature profile is observed to be strongly flattened near the axis. The electron temperature gradient only follows the ETG mode threshold near the leading edge of the transport barrier [4-2]. The cause of this flattening is not understood. Thus, the predicted peaking of the electron temperature within the ITB should not be taken as definitive.

Table 4-1

SUMMARY OF TRANSPORT RESULTS

\begin{tabular}{|c|c|c|c|c|c|}
\hline & & $\begin{array}{l}13.2 \mathrm{MA} \\
\text { Original }\end{array}$ & $\begin{array}{c}13.2 \mathrm{MA} \\
\mathrm{N}_{\mathrm{eG}}\end{array}$ & $\begin{array}{c}\text { 8.27 MA } \\
\text { BASE }\end{array}$ & $\begin{array}{c}8.27 \mathrm{MA} \\
\text { ITB }\end{array}$ \\
\hline$\overline{I_{p}}$ & $M A$ & 13.15 & 13.15 & 8.27 & 8.27 \\
\hline$l_{\text {boot }}$ & $M A$ & 10.00 & 8.07 & 6.70 & 7.94 \\
\hline$I_{N B I}$ & MA & 0.59 & 1.87 & 1.42 & 1.47 \\
\hline Iohm & MA & 2.55 & 3.2 & 0.147 & -1.15 \\
\hline$I_{\text {boot }} / l_{p}$ & $\%$ & 76 & 61 & 81 & 96 \\
\hline $\mathrm{N}_{\mathrm{e}}$ & $10^{20} / \mathrm{m}^{3}$ & 5.03 & 1.95 & 1.94 & 1.96 \\
\hline \multicolumn{2}{|c|}{$\mathrm{N}_{\mathrm{e}} / \mathrm{N}_{\mathrm{e}} \mathrm{G} w$} & 2.29 & 0.89 & 1.40 & 1.41 \\
\hline $\mathrm{Pa}$ & MW & 1,713 & 724 & 434 & 176 \\
\hline$P_{\text {rad }}$ & MW & 280 & 280 & 271 & 217 \\
\hline$\tau_{e}$ & sec & 0.51 & 1.36 & 1.10 & 1.98 \\
\hline $\mathrm{H}_{89 \mathrm{p}}$ & & 1.23 & 2.38 & 2.26 & 3.03 \\
\hline$\beta_{p}$ & & 2.51 & 2.93 & 3.75 & 3.40 \\
\hline$\beta_{T}$ & $\%$ & 6.65 & 7.78 & 4.03 & 3.66 \\
\hline $\mathrm{Nd} / \mathrm{Nt}$ & $\%$ & 50 & 50 & 50 & 90 \\
\hline
\end{tabular}

Common parameters: $\mathrm{Bt}=7.98 \mathrm{~T}, \mathrm{R}=5.52 \mathrm{~m}, \mathrm{a}=1.38 \mathrm{~m}, \mathrm{P}_{\mathrm{NBI}}=50 \mathrm{MW}$, elongation $=1.8$.

The GLF23 model predicts the energy confinement of the ITB case to be three times L-mode scaling (ITER 89p). The high $\mathrm{q}$ in the core of this lower current equilibrium deteriorates the ion neoclassical energy confinement. Higher confinement within the ITB should be possible at lower $\mathrm{q}$ and with a flatter $\mathrm{q}$ profile.

The deuterium/tritium ratio is unacceptably high (90\%) and the line average density is still above the Greenwald limit $\left(\mathrm{n}_{\mathrm{e}} / \mathrm{n}_{\mathrm{eGW}}=1.41\right)$. 

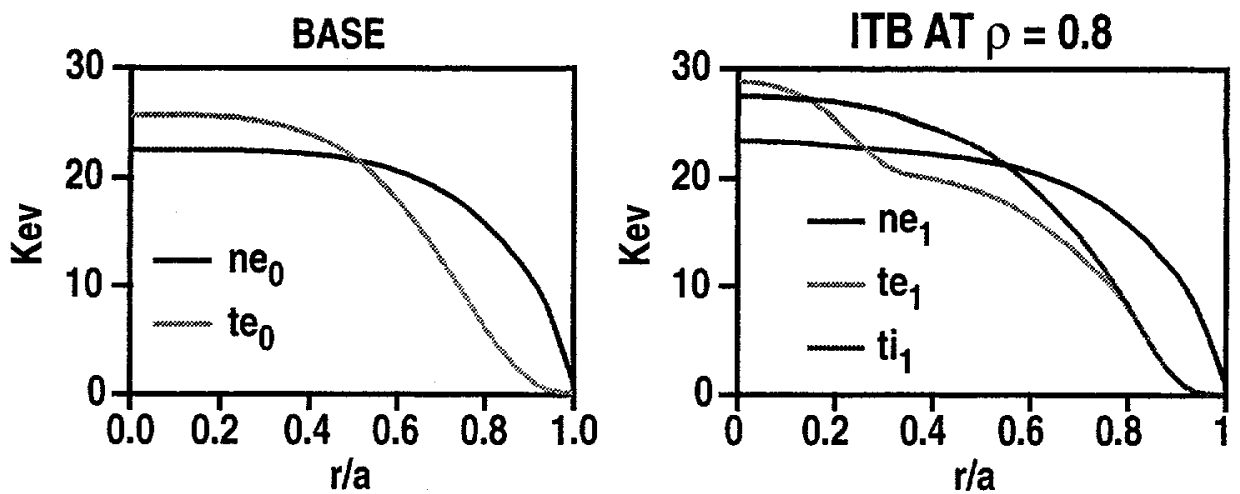

Fig. 4-1. Note that $T_{i}=T_{e}$ for the base case. The GLF23 transport model was used for ITB case.
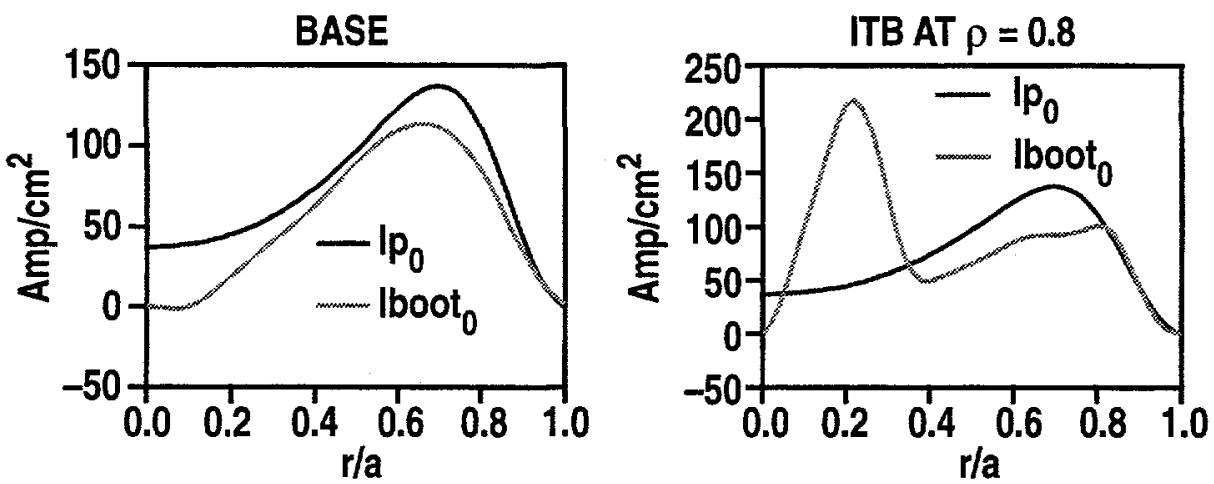

Fig. 4-2. $I_{\text {boot }} / I_{p}=81 \%$ for the base case and $I_{\text {boot }} / I_{p}=96 \%$ for the ITB case.

\subsection{FUTURE DIRECTIONS}

The bootstrap alignment of the TOQ equilibrium is near optimum and cannot be expected to be much improved by the self-consistent transport modeling. Iteration between TOQ and ONETWO can get close to a final design point with an energy confinement time near to that determined by GLF23. It is highly desirable to find a starting equilibrium with:

$\mathrm{I}_{\text {boot }} / \mathrm{I}_{\mathrm{p}}>80 \%$

$\mathrm{H}_{89 \mathrm{p}^{\sim 3}}$ consistent with ONETWO calculation of the fusion power

$\mathrm{n}_{\mathrm{e}} \sim$ Greenwald density

In the above cases we reported on an attempt to move towards this design point by lowering the plasma current. This is not the optimum direction to move. The following argument suggest that lowering the minor radius would be a better direction. The Greewald density limit is $I / \pi a^{2}$. The TOQ equilibrium uses $T=p^{\sigma}, n=p^{(1-\sigma)}(\sigma=0.8$ for 
the cases in Fig. 4-1). It was found that the bootstrap current goes up with $\mathrm{n} / \mathrm{T}$ for a given pressure profile. The ratio $\mathrm{n} / \mathrm{T}$ scales like

$$
\mathrm{n} / \mathrm{T} \sim \mathrm{n}^{2} / \beta \mathrm{B}^{2} \sim \mathrm{f}_{\mathrm{GW}}(\mathrm{I} / \mathrm{aB})^{2} / \mathrm{Ba}^{2} .
$$

Thus, at fixed $\beta$ and $q \sim \mathrm{I} / \mathrm{aB}$ it is necessary to reduce the size a to get to higher $\mathrm{n} / \mathrm{T}$. Lowering the current alone lowers $n / T$. In order to take full advantage of the high energy confinement predicted by GLF23 for a reactor with an ITB, while maintaining a high bootstrap fraction, it is recommended that that a smaller minor radius design point be considered.

\section{REFERENCES}

[4-1] R.E. Waltz et al., Phys. Plasmas 4, (1997), 2482.

[4-2] B.W.Stallard et al., to be published in Phys. Plasmas. 


\section{ECCD CONSIDERATIONS FOR ARIES-AT}

\subsection{INTRODUCTION}

Electron cyclotron waves were not considered for auxiliary heating and current drive in previous ARIES power plant studies because of concern about the low efficiency of the gyrotron sources and other technological issues. The current drive efficiency also tends to be low compared to other rf schemes, such as fast waves and lower hybrid waves. However, recent advances in the EC technology and experimental data base merit a reexamination of the possible roles EC waves can play in ARIES-AT.

Significant improvements in the performance of gyrotrons and windows in the millimeter-wave range have recently been reported [5-1]. With the use of single-stage depressed collectors and new window materials, the gyrotron efficiencies in the $50 \%$ range have been recorded. For example, FZK Karlsruhe reported a $51 \%$ efficiency at $140 \mathrm{GHz}, 460 \mathrm{~kW}$ and a pulse length of $0.2 \mathrm{~s}$, while at the same frequency a $49 \%$ efficiency is achieved by GYCOM at $800 \mathrm{~kW}, 0.6 \mathrm{~s}$ pulse, both using single-stage depressed collectors. At GYCOM, a $65 \%$ efficiency was recorded at $110 \mathrm{GHz}, \mathrm{TEM}_{00}$ output mode, $1 \mathrm{MW}$ of power and $0.1 \mathrm{~ms}$ pulse length [5-2]. At the same time, transmitted power levels have been raised substantially with the use of new window materials such as silicon, sapphire and diamond that allow for cyrogenic edge cooling. At this stage, a transmitted power density in excess of $300 \mathrm{MW} / \mathrm{m}^{2}$ appears to be well within reach.

In the past years significant results have been achieved in EC current drive experiments. On DIII-D, off-axis current drive by EC waves was demonstrated for the first time [5-3]. Variation of the location of the driven current by steering the EC beam off reflecting mirrors has also been demonstrated. These developments add to the confidence of the use of EC waves for localized current profile control.

There are other characteristics of EC waves that merit their consideration in the ARIES-AT fusion power plant. In the frequency range of operation they do not interact with thermal ions and energetic alpha particles, thus eliminating a source of complication in reactor applications. Coupling of the wave energy into the plasma is relatively simple because the EC waves readily propagate in free space, and wave launching often requires only a small hole (series of holes) in the first wall. EC waves can also be considered for breakdown and preionization, and central heating and current profile control during startup. 
Nevertheless, there are potential issues related to ECCD in a reactor grade plasma. Penetration to the plasma core may be limited due to density cuttoffs for both the $\mathrm{O}$ - and $\mathrm{X}$-modes; however, as long as the dielectric constant, $\left(\omega_{\mathrm{pe}} / \omega_{\mathrm{ce}}\right)^{2}$, is below unity throughtout the plasma, which is the case for ARIES-AT, this should not be an issue. The CD efficiency is likely to be modest compared to other rf techniques, especially LH waves, making it more appropriate as a method for localized current profile control, e.g., to stablize the neoclassical tearing mode. However, in an operating scenario where only a small amount of seed current needs to be driven (e.g., $<0.5 \mathrm{MA}$ ), EC waves should be considered in order to take advantage of the simple launching scheme and its engineering compatibility with the rest of the fusion power core.

\subsection{ECCD CALCULATIONS}

The possibility of using EC waves for on-axis and off-axis seed current drive has been explored in the context of ARIES-AT. A series of calculations based on the use of the O-mode has been performed using the TORAY ray tracing code in conjunction with the ONETWO transport code. The analysis has been done in the snapshot mode. For this purpose, an equilibrium in the EQDSK format (g099326) has been used, which has the following global parameters: $R_{0}=5.52 \mathrm{~m}, \mathrm{a}=1.38 \mathrm{~m}, \mathrm{~B}_{0}=7.98 \mathrm{~T}, \mathrm{I}_{\mathrm{p}}=11.2 \mathrm{MA}, \beta=5.53 \%$, $\beta_{\mathrm{N}}=5.44, \mathrm{~T}_{\mathrm{eo}}=28.25 \mathrm{keV}$, and $\mathrm{n}_{\mathrm{eo}}=3.42 \times 10^{20} \mathrm{~m}^{-3}$. The wave frequency is set at $204 \mathrm{GHz}$, which corresponds to the $\mathrm{f}=\mathrm{fce}$ resonance surface being located near the magnetic axis, at $\mathrm{R}=5.83 \mathrm{~m}$ where $\mathrm{B}=7.29 \mathrm{~T}$.

For on-axis drive, a typical scenario involves the $\mathrm{EC}$ beam being launched from the outboard edge along the equatorial plane, at an angle of $10^{\circ}$ off the radial direction. With this launch scheme, the wave energy is absorbed within $\rho=0.2$ in a single pass, as shown in Fig. 5-1. The bulk of the wave power is absorbed before the five rays that represent the beam traverse the resonance surface. The calculated current drive efficiency is $0.004 \mathrm{~A} / \mathrm{W}$, corresponding to a normalized efficiency $\gamma$ of 0.05 , which is modest. For a typical on-axis seed current of $0.2 \mathrm{MA}$, about $50 \mathrm{MW}$ of EC power will be required. It is suspected that, in this case, oppositely directed currents may have been generated leading to a low net driven current. Further detailed exploration with the launch scheme will be needed to improve on the $\mathrm{CD}$ efficiency.

In the case of off-axis drive, the target location of current drive is $\rho \sim 0.8$, where shear reversal typically occurs. By maintaining the same launch scheme as the on-axis drive case but varying the incident beam direction in the equatorial plane, it is possible to move the driven current outward from the magnetic axis. At an angle of $28^{\circ}$ off the radial 
direction, the driven current is found to peak at $\rho \sim 0.8$, the desired location. The result of this calculation is displayed in Fig. 5-2, with en efficiency of $0.02 \mathrm{~A} / \mathrm{W}$, which requires $\sim 40 \mathrm{MW}$ to drive a typical off-axis seed current of $0.8 \mathrm{MA}$. The reason for this rather good efficiency is because of the local relatively low density and high temperature, and no cancellation of currents, even though the wave power is absorbed at a location where trapped electron degradation of the $\mathrm{CD}$ efficiency is quite strong.

(a)
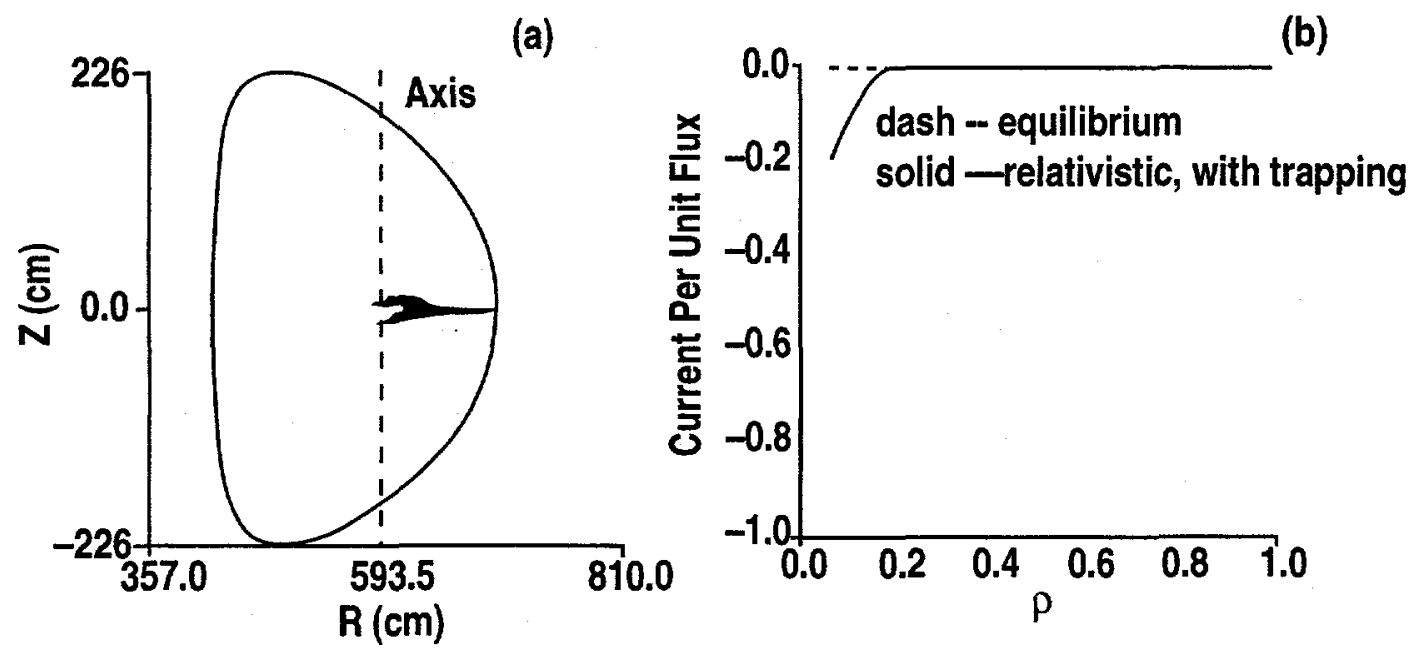

Fig. 5-1. Calculated on-axis current drive with EC waves in typical ARIES-AT equilibrium: (a) ray trajectories, and $(b)$ driven current profile.

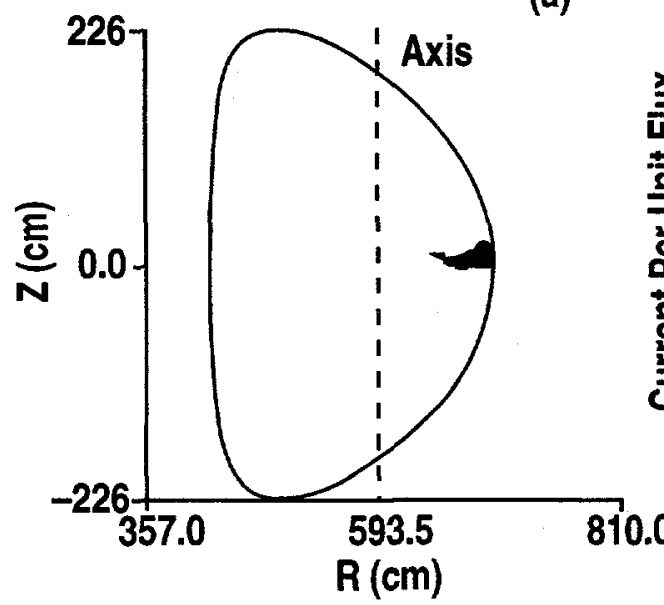

(a)

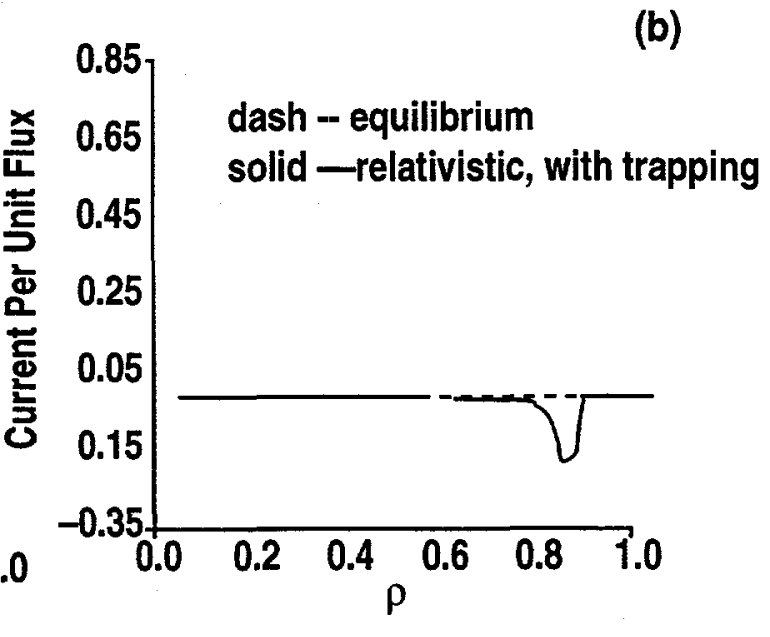

Fig. 5-2. Calculated off-axis current drive with EC waves in typical ARIES-AT equilibrium: (a) ray trajectories, and $(b)$ driven current profile. 


\subsection{DISCUSSIONS}

Based on the initial calculations reported here, one can conclude that it is possible to devise EC waves to drive on-axis and off-axis currents in a typical ARIES-AT equilibrium by using a single frequency and $\mathrm{O}$-mode launch from the outboard midplane. However, the case studies so far show the on-axis drive to be inefficient and further analysis needs to be carried out to improve on it. On the other hand, the off-axis scenarios that have been examined appear to have acceptable efficiencies for application in ARIESAT. Launching from off the outboard midplane has been examined but has so far not identifed scenarios more efficient than the off-axis drive results reported here.

The need for rotation generation for kink stabilization will likely entail tangential injection of neutral beams, which can be configured to drive the bulk of the off-axis seed current. In this scenario, ECCD can play a complementary role of profile control in the vicinity of the shear reversal region and for stabilizing the neoclassical tearing mode. These issues should be further investigated in the ARIES-AT project during FY00.

\section{REFERENCES}

[5-1] M. Thumm, "Recent Development of High Power Gyrotrons and Windows for EC Wave Applications," Proc. 12th Top. Conf. on Radio Frequency Power in Plasmas, Savannah, GA (1997) 183; also AIP Conf. Proc. 403.

[5-2] M. Thumm, "State-of-the-Art of High-Power Gyro-Devices and Free Electron Masers Update 1996,” FZKA Report 5877, FZK Karlsruhe, February 1997.

[5-3] T. Luce, et al, "Current Profile Modification with Electron Cyclotron Current Drive," Bull. Am. Phys. Soc. 43 (1998) 1772. 


\section{DIVERTOR-RELATED ISSUES FOR ARIES-AT}

\subsection{INTRODUCTION}

A major worry in designing the ARIES-AT tokamak is high heat loading on its divertor structure. We quantify the severity of this problem for the ARIES-AT divertor in Section 6.2 and discuss ways in which this problem might be ameliorated. Additional divertor-related issues that can affect advanced tokamak performance have arisen recently and are addressed in Section 6.3. These include the sensitivity of the core and divertor plasmas to slight variation in magnetic balance and the effectiveness of the slot divertor in reducing heat flux outside the slot. In Section 6.4, we summarize our results and propose direction for future work.

\subsection{ESTIMATION OF THE PEAKED HEAT FLUX INSIDE THE VESSEL}

The total heat flux is composed of two sources: (1) direct particle heating of the divertor surfaces, and (2) the heating due to (electromagnetic) radiated power. After a brief description of how each of these heat flux components is evaluated, evaluate the heat flux inside the ARIES-AT vessel under different scenarios for heat exhaust.

\subsubsection{Particle Heating in the Divertors}

We assume that the radial profile of the heat flux in the scrape-off layer (SOL) has an exponential form. An expression for the peak heat flux at the divertor can be written:

$$
\mathrm{Q}_{\mathrm{div}, \mathrm{S}}=\frac{\mathrm{P}_{\text {heat }} \times\left(1-\mathrm{f}_{\mathrm{rad}}\right) \times \mathrm{f}_{\text {outboard/total }} \times \mathrm{f}_{\nabla \mathrm{B} / \text { total }} \times\left(1-\mathrm{f}_{\mathrm{pfr}}\right) \times \sin (\alpha)}{2 \pi \times \mathrm{R}_{\mathrm{S}} \times \mathrm{f}_{\exp } \times \lambda_{\mathrm{p}} \times\left[\frac{\mathrm{f}_{\text {exp }} \times \lambda_{\mathrm{p}}}{\mathrm{R}_{\mathrm{S}}}\right]}
$$

where

$Q_{\text {div,S }}$ is the peak heat flux at the divertor strike point,

$P_{\text {heat }}$ is the total heating power,

$R_{S}$ is the major radius of the divertor strike point, $R \geq R_{S}$,

$\lambda_{\mathrm{p}}$ is the midplane heat flux scrape-off length,

$f_{\text {exp }}$ is the flux expansion at the divertor target,

$\alpha$ is the angle between the divertor incline and the separatrix,

$\mathrm{f}_{\mathrm{rad}}$ is the ratio of total radiated power to total input power, 
$f_{\text {outboard/total }}\left(f_{\text {inboard/total }}\right)$ is the ratio of power flowing into the outboard (inboard) SOL to the total power flowing into the SOL.

$f_{\nabla \mathrm{B} / \text { total }}$ is the ratio of power striking the outboard divertor in the $\nabla \mathrm{B}$ direction to the total power striking both upper and lower outboard divertors, $\mathrm{f}_{\mathrm{pfr}}$ is the fraction of power flowing into the private flux region.

The peak heat flux predictions of Eq. (1) have been compared with DIII-D data and generally found to be within $20 \%$ of the measured peak heat flux at the outboard divertor strike point in attached plasmas.

\subsubsection{Radiated Power From Electromagnetic Sources}

An estimate of the poloidal heat flux distribution along the first wall of the vacuum vessel, given the radiated power source function, can be evaluated using the RADLOAD program. The RADLOAD program assumes toroidal symmetry.

RADLOAD follows a "multifilament" approach, where the radiating heat flux (QEM) onto a wall surface element from a radiating source distribution is given by:

$$
\mathrm{Q}_{\mathrm{EM}}=\int_{\mathrm{V}} \varepsilon \overrightarrow{\mathbf{r}} \cdot \hat{\mathrm{n}} /\left[4 \pi \mathrm{r}^{3}\right] \mathrm{dV}
$$

where

$\varepsilon$ is the emissivity of the filament

$\vec{r}$ is the vector from the wall segment to the radiating filament

$\hat{n}$ is the unit normal vector out of the wall surface element.

The above integral is performed over that part of the radiating volume visible to the surface element. To do this, the radiating region is divided into "filaments" extending around the machine in the toroidal direction with each filament denoted by its own set of poloidal coordinates. The limits of integration for each filament are determined by testing whether the line-of-sight along the filament is visible to the wall element in question. The heat flux QEM is found by summing over the contribution of each filament.

\subsubsection{Application to Total Heat Flux}

The base case parameters used in the calculations below are given in Table 6-1. Since there are no detailed drawings of the ARIES-AT divertor available at present, the shape and dimensions for the divertor geometry are based on ARIES-RS data [6-1]. The 
divertor legs in this design are inclined at an angle $(\alpha)$ of $10^{\circ}$ relative to the divertor surfaces in the slot in order to increase the effective wetted area and minimize the heat flux. Because much of the physics in the boundary and scrape-off layer (SOL) regions of highly triangular, double-null (DN) divertors is not well known, the selection of the parameters that should be used in Eq. (1) needs to be considered carefully. Guidance that might be provided by the UEDGE divertor transport modeling code is unavailable until upgrades are implemented (e.g., including the symmetry-breaking $\nabla \mathrm{B}_{\mathrm{T}}$ drifts in doublenull and near double-null geometries). Instead we must rely on extrapolations of available experimental data in choosing "reasonable" values for the required input. For example, the value of $f_{\text {outboard/total }}$ in Table 6-1 comes from heat flux measurements in a series of experiments in DIII-D for which $\mathrm{H}$-mode DN plasmas with triangularity similar to ARIES-AT was found to have a ratio of outboard-to-inboard power flow of $\geq 4: 1$. We take the power scrape-off length $\left(\lambda_{\mathrm{p}}\right)$ at the outboard midplane as $1 \mathrm{~cm}$. About $10 \%$ of the power flow to the divertors spills into the private flux region (i.e., $\mathrm{f}_{\mathrm{pfr}} \approx 0.1$ ), again based on analyses of single-null (SN) and double-null infrared camera data. The parameters used in Eq. (1) are summarized in Table 6-1.

Table 6-1

ARIES-AT Parameters

\begin{tabular}{lcc}
\hline & Inboard Leg(s) & Outboard Leg(s) \\
\hline Pheat $(M W)$ & 513 & 513 \\
$f_{\text {rad }}$ & 0.18 & 0.18 \\
$\gamma_{\mathrm{p}}(\mathrm{cm})$ & 1.0 & 1.0 \\
$\mathrm{f}_{\text {exp }}$ & 5 & 5 \\
$\mathrm{f}_{\text {pfr }}$ & 0.1 & 0.1 \\
$\mathrm{R}_{\mathrm{S}}(\mathrm{m})$ & 3.86 & 4.80 \\
fract of SOL power flow & 0.2 & 0.8 \\
$\mathrm{f}_{\nabla \mathrm{B} / \text { total }}$ & 0.5 & 0.5 \\
$\alpha\left({ }^{\circ}\right)$ & 10 & 10 \\
$\mathrm{Q}_{\text {div }, \mathrm{S}}\left(\mathrm{MW} / \mathrm{m}^{2}\right)$ & 5.4 & 17.3 \\
\hline
\end{tabular}


We estimate the peak heat flux under the inboard and outboard divertor legs by assuming that the radiated power from the scrape-off layer and divertors are negligible. These estimates represent an upper bound on the peak heat flux that can be expected for ARIES-AT ("worst case"). Using the parameters from Table 6-1, we estimate that the peak heat flux under the inboard legs is $\approx 5.4 \mathrm{MW} / \mathrm{m}^{2}$ and the peak heat flux under the outboard legs is $\approx 17.3 \mathrm{MW} / \mathrm{m}^{2}$.

This value of the peak heat flux under the outboard legs may exceed tolerable limits of what might be safely handled (e.g., $\approx 10 \mathrm{MW} / \mathrm{m}^{2}$ ). Enhancing radiated power would lower this heat flux. Two approaches are commonly discussed in this regard: (1) "radiating divertor" and (2) "radiating mantle." While the complexities and uncertainties involved in achieving each of these operating modes is beyond the scope of this present study, we examine the appropriateness of either approach as applied in an ARIES-AT setting, e.g., the location and magnitude of a radiating source impacts the poloidal heat flux distribution and hence its cooling requirements.

For example, suppose it is desirable to reduce the peak particle heat flux $\mathrm{Q}_{\operatorname{div}}, \mathrm{S}$ at the outboard strike points to $10 \mathrm{MW} / \mathrm{m}^{2}$. This would require that $53 \%$ of the heating power be radiated (somewhere). Under a "radiating divertor" scenario similar to the one discussed for ARIES-RS [6-2], 18\% of the total heating power would be radiated from the core (mostly bremsstrahlung radiation) and the additional $35 \%$ of the radiated power would be evenly divided between the two divertors. RADLOAD has modeled the radiated power heat flux distribution with 150 individual source radiators arranged uniformly between the outboard separatrix and the $1 \mathrm{~cm}$ flux surface bounded by the divertor floor and the $\mathrm{X}$-points. The analysis indicates that the radiative component would add significantly $\left(\approx 2.3 \mathrm{MW} / \mathrm{m}^{2}\right)$ to the heat flux near the outboard strike points. Consequently, the total heat flux (i.e., Qdiv,S + QEM) would actually be more than $12 \mathrm{MW} / \mathrm{m}^{2}$, and the radiating component itself would be a significant contributor to divertor heating.

The "radiating mantle" approach assumes that the source of the radiated power comes predominantly from edge of the main plasma. As in the "radiating divertor" case described above, lowering $q_{\mathrm{div}, \mathrm{S}}$ to $10 \mathrm{MW} / \mathrm{m}^{2}$ would also require that $53 \%$ of the heating power be radiated, although here most of the radiated power would be coming from this edge region of the main plasma (i.e., the "mantle"). RADLOAD models the "radiating mantle" using 300 individual source radiators arranged poloidally uniformly around the core plasma immediately inside the separatrix. The radiative heat flux $\left(\mathrm{Q}_{\mathrm{EM}}\right)$ over most of the outboard-side vacuum vessel wall is $\approx 0.8 \mathrm{MW} / \mathrm{m}^{2}$ and over most of the inboard wall is $\approx 0.5 \mathrm{MW} / \mathrm{m}^{2}$. While the radiative heating on that part of the divertor 
lying directly under the $\mathrm{X}$-points can be relatively high $\left(\approx 0.6 \mathrm{MW} / \mathrm{m}^{2}\right)$, the $\mathrm{Q}_{\mathrm{EM}}$ contribution inside the slots is much less $\left(<0.2 \mathrm{MW} / \mathrm{m}^{2}\right)$. Hence, the radiated power contribution to the total heat flux would not add appreciably to the locations where particle heating $\mathrm{Q}_{\mathrm{div}, \mathrm{S}}$ would be high (i.e., inside the slots). Furthermore, the radiated power would be spread fairly uniformly around the interior of the vacuum vessel. Thus, from the standpoint of power handling, a "radiating mantle" solution would be preferential to the "radiating divertor" solution.

\subsection{ADDITIONAL ISSUES}

Two other issues can set constraints on what may ultimately be necessary for successful divertor operation of ARIES-AT. The first issue has to do with shape control, or more specifically, how well the magnetic balance can be maintained. The second issue is the effectiveness of a slot divertor, given the likelihood of elevated ion temperatures in the SOL.

\subsubsection{Magnetic Balance of the Double-Null}

Recent experiments have shown that it is crucial that the shaping control be able to maintain magnetic balance to within specified tolerances, if a magnetically-balanced DN configuration is desired [6-3]. To quantify "magnetic balance," we introduce a parameter drSEP, defined as the distance between the upper divertor separatrix and the lower divertor separatrix, as determined by the radial distance at an outboard midplane location (See the inset to Fig. 6-1). This definition implies that the plasma equilibrium for a "positive" value of drSEP is biased toward the upper divertor and that the equilibrium for a "negative" value of drSEP is biased toward the lower divertor. In the following, the $\nabla B$-drift will be toward the lower divertor.

Figure 6-1, which plots the peak heat flux "asymmetry" as a function of drSEP for a set of DIII-D data, illustrates that heat flux balance is sharply dependent on drSEP near magnetic balance (i.e., drSEP $=0$ ). This means, for example, that for a nearly magnetically-balanced DN divertor a variation of about one power scrapeoff length $(\approx$ $0.6 \mathrm{~cm}$ in DIII-D), the ratio of peak divertor heat fluxes changes from 50-50 (heat flux balance) to 90-10. Consequently, if balancing the heat flux in a DN geometry in ARIES-AT is desired, control over drSEP must be maintained to better than one power scrape-off length (i.e., $<0.5-1.0 \mathrm{~cm}$ ). 


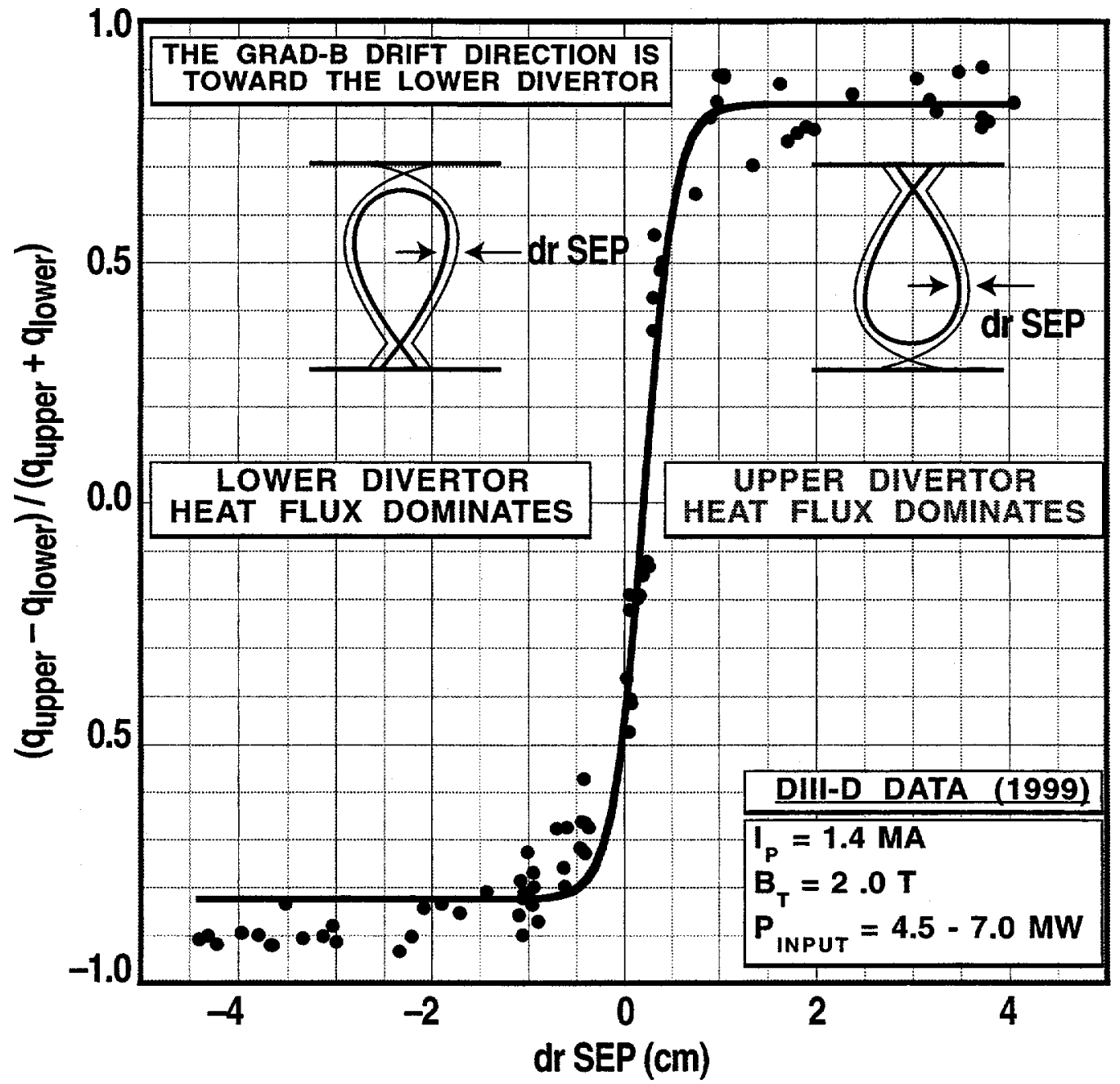

Fig. 6-1. The peak heat flux delivered to each DIII-D divertor is a sensitive function of drSEP, when drSEP is near zero. A positive value of drSEP indicates that the plasma is biased toward the upper divertor, as shown in the inset; a negative value of drSEP indicates that the configuration is biased toward the lower divertor. The direction of the $\nabla B$ drift is toward the lower divertor. qupper and qlower are the peak heat flux values measured in the upper and lower divertors, respectively. In order to balance qupper and qlower, note that there has to be a slight bias in drSEP toward the upper divertor $(\approx 0.2-0.3 \mathrm{~cm})$.

Magnetic balance also affects plasma performance in other ways. For example, the $\mathrm{H}$-mode density limit (i.e., the density at which an H-L back transition occurs) is measurably lower in unbalanced DNs biased away from the $\nabla \mathrm{B}$ drift direction. As with the above heat flux balance results, the greatest change in the back transition density also occurs near drSEP $=0$, more specifically between 0 and $+1 \mathrm{~cm}$, i.e., between a magnetically balanced DN and a DN biased slightly toward the upper divertor. Thus, an ARIES-type tokamak operating at higher density (e.g., near the Greenwald density limit) 
may be vulnerable to losing good $\mathrm{H}$-mode confinement if drSEP "slips" from magnetic balance to slightly unbalanced in the direction opposite the $\nabla \mathrm{B}$ drift direction.

Finally, control over magnetic balance is important even during the early stages of the discharge, if the heating power used is near that required for the L-H transition. Under these conditions, a "slide" in the magnetic balance from DN to a DN slightly biased in a direction opposite the $\mathrm{\nabla B}$ drift results in a loss of $\mathrm{H}$-mode confinement. This behavior in DIII-D has occurred for drSEP values between 0 and $+1 \mathrm{~cm}$, although it has not been observed when drSEP "slides" the other way (i.e., 0 toward $-1 \mathrm{~cm}$ ). The fact that it is easier to maintain $\mathrm{H}$-mode confinement when the $\nabla \mathrm{B}$ drift is toward the principal $\mathrm{X}$-point is not surprising. The significant degradation in confinement when drSEP changes from 0 to $+1 \mathrm{~cm}$ is another indication that control of drSEP near the magnetic balance configuration is a very important consideration.

\subsubsection{Heat Flux Reduction Outside the Slot Divertors}

The "slot divertor" concept offers several advantages in pumping and heat flux management. For example, slotted divertors, as envisioned by ARIES-AT and other advanced tokamaks, may allow injected impurity ions to radiate away a significant amount of incoming power by facilitating a buildup ("enrichment") of impurities in the divertor while simultaneously inhibiting their escape into the main plasma. Experiments related to this concept are under active investigation, e.g., "Puff and Pump" [6-4].

Yet, in making this assessment of how a "radiating divertor" might function in an ARIES-AT environment, it is important to determine how heat flux outside the slot is affected during radiative cooling. For example, one common way to reduce divertor heating is to inject deuterium gas, which cools the divertor electron temperatures and raises the divertor electron density, both of which raise the hydrogenic- and impurity(carbon) radiated power in the divertor. Eventually, the plasma "detaches" from its divertor strike points, triggering a partially detached (or strongly radiating) divertor (PDD) condition. In Fig. 6-2(a), a "slot-like" DIII-D divertor configuration with high triangularity is shown with a $1.5 \mathrm{~cm}$ flux surface (as measured from the outboard midplane) intersecting the baffle or "slot" roof. During neutral deuterium gas puffing, the peak heat flux inside the slot was reduced by a factor of 3-4 from its original value. Yet, the heat flux at the entrance to the slot (approximately two power scrapeoff lengths outside the separatrix) was unchanged [Fig. 6-2(b)]. Hence, one may not assume that the heat flux outside a slot is reduced commensurate with the heat flux inside the slot during radiating divertor conditions. 


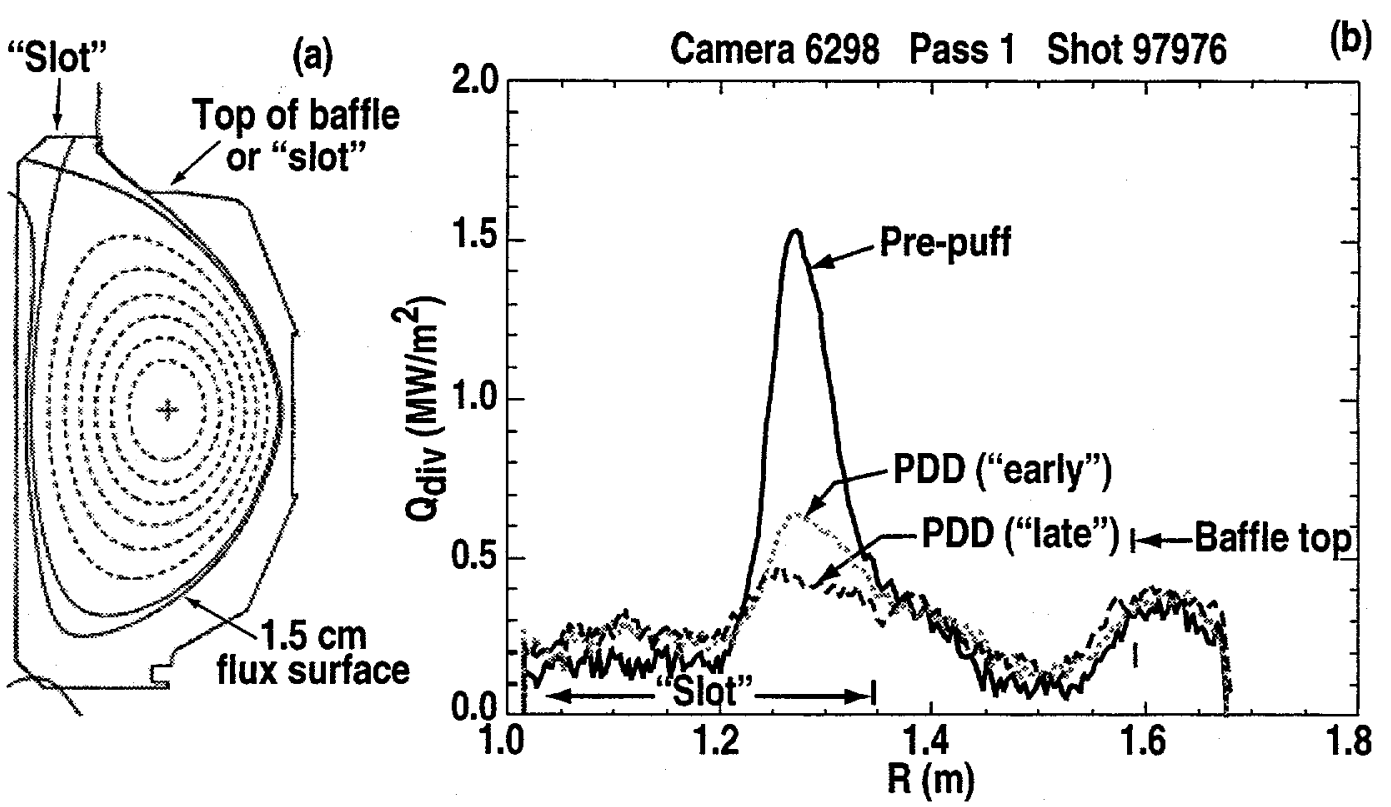

Fig. 6-2. (a) The $1.5 \mathrm{~cm}$ flux surface (as measured from the outboard midplane) intersects the baffle roof. For DIII-D, the $1.5 \mathrm{~cm}$ surface is approximately twice the power scrape-off length. (b) While the heat flux inside the "slot" was reduced significantly during deuterium gas puffing, the heat flux at the entrance to the slot was unchanged. The solid line represents the heat flux profile prior to gas deuterium gas injection, the dotted curve after about $400 \mathrm{~ms}$ of gas injection ("early" PDD), and the dashed curve after about $1400 \mathrm{~ms}$ of gas injection ("late" PDD).

The reason for this resistance to heat flux reduction on the outside slot structure is under investigation. It has been argued that the power lost through the electron channel is responsible for the peaked heat profile near the separatrix strike point prior to introducing a radiating divertor. Since electrons can be efficiently cooled by enhancing the radiation in the divertor, the peak heat flux found under the separatrix strike points can be reduced significantly using radiative divertor approaches. "Deep" into the SOL, however, the ions may become the principal contributor to the heat flux, since the ion temperature scale length in the SOL is much larger than the electron temperature scale length. While radiated power enhancement in the SOL and divertors may be effective for cooling the electrons, cooling energetic ions once they are in the SOL is much more challenging. Because of the relatively low particle density in the SOL, energy exchange between cold electrons and warm ions is not an efficient way to bring down the ion temperature.

Because of this inefficient energy exchange between electrons and ions in the SOL, the energetic ions in the SOL not only make reducing the heat flux on the "top" of a slotted divertor difficult, but also make the issue of physical sputtering on the structure itself problematical. In the latter, an important variable is the slot material. For example, tungsten may be a viable divertor material if the ion temperature at the plasma/material 
interface can be kept under $50 \mathrm{eV}$ [6-5,6-6]. This would imply a relatively cool edge plasma $(<100 \mathrm{eV}$ at the midplane, based on simple one-dimensional transport analysis [6-7] along the separatrix field line and on $T_{e} \approx T_{i}$ ).

\subsection{Summary and Conclusions}

If $18 \%$ of the total plasma heating power is radiated from the main plasma, then the peak heat flux is found at the outer strike points $\left(\approx 17 \mathrm{MW} / \mathrm{m}^{2}\right)$. By increasing the total radiated power fraction in the core to a little over 0.5 , the peak heat flux under the outboard divertor legs would be lowered to a more manageable $10 \mathrm{MW} / \mathrm{m}^{2}$. In terms of dissipating the power flowing out of the core plasma under these conditions, we think that a "radiating mantle" is preferable to a "radiating divertor"; for ARIES-AT steady-state operating conditions, the latter would result in about $20 \%$ less heating of vulnerable areas near the outboard separatrix strike points. The possibility of reducing the peak heat flux even further $\left(\approx 6 \mathrm{MW} / \mathrm{m}^{2}\right)$ was explored previously by using a radiating mantle/divertor, although achieving this level required elevated values of impurity enrichment in the divertors [6-2].

While progress has been made in addressing aspects of the heat flux handling problems, new issues have arisen. Experimentally, we have found that the heat flux balance between divertors (and several core plasma properties) are very sensitive to how accurately the double-null can maintain magnetic balance. These results suggest the importance of the plasma shaping system for ARIES-AT to be able to control drSEP to better than about $\pm 0.5 \mathrm{~cm}$ tolerance. As a precaution against a loss in control of heat flux balance, each divertor should be able to withstand heating from single-null shapes, at least for a short time. The peak heat flux under this operating scenario would raise the peak heat flux at one of the divertors to $\approx 29 \mathrm{MW} / \mathrm{m}^{2}$. In addition, the typically broad ion temperature profile in the SOL can subject the area next to the slot to "radiation-resistant" heat flux, as well as energetic ion bombardment causing structural erosion.

The divertor heating issues and the problems related to hot ions in the SOL and divertor suggest a common solution - a "radiating mantle." If a significant amount of the power input is radiated in the plasma mantle, then (1) not only is the peak heat flux reduced, but there is less power flowing into the SOL, making a loss of drSEP control less disastrous, and (2) the ion temperature can be more effectively lowered, since the higher radiated power and higher density inside the main plasma cools the electrons and ultimately the ions via strong electron-ion coupling. 
The main difficulty, however, in using the "radiating mantle" approach (or some combination of "radiating mantle" and "radiating divertor") is the uncertainty introduced to the core plasma transport by the radiating layer. In advanced tokamaks, such as ARIES-AT, the transport barrier and peak in the bootstrap are usually located out near the plasma edge (e.g., $\rho / a \approx 0.8$ for ARIES-AT). Further work in this area should focus on the conditions under which a radiating mantle and transport barrier might co-exist.

\section{REFERENCES}

[6-1] M.S. Tillack and the ARIES Team, "Engineering Overview of ARIES-RS Tokamak Power Plant," Presented at the 19th Symposium on Fusion Technology, Lisbon, Portugal, 16-20 September 1996.

[6-2] S.C. Jardin, et al., "Physics Basis for a Reversed Shear Tokamak Power Plant," to be published in Fusion Engineering and Design.

[6-3] T.W. Petrie, et al., "A Comparison of Plasma Performance Between Single-Null and Double-Null Configurations During ELMing H-mode," Presented at the 26th European Physical Society Conference on Controlled Fusion and Plasma Physics, Maastrict, The Netherlands, 14-18 June 1999.

[6-4] M.R. Wade, et. al., "Demonstration of the ITER Power Exhaust Solution Using the "Puff and Pump" Technique in DIII-D," Presented at the 26th European Physical Society Conference on Controlled Fusion and Plasma Physics, Maastrict, The Netherlands, 14-18 June 1999.

[6-5] W. Eckstein and J. Laszlo, Journal of Nucl. Mater., 183 (1991) 19.

[6-6] C.H. Wu and U. Mszanowski, Journal of Nucl. Mater., 218 (1995) 293.

[6-7] W.L. Barr and B.G. Logan, Fusion Technology, 18 (1990) 251. 


\section{CURRENT AND STORED ENERGY RAMPUP IN THE ARIES-AT TOKAMAK}

\subsection{MODEL EQUILIBRIUM}

The work was done using Politzer's TD spreadsheet (v. 10.07). This is a 0-D tokamak simulation, which includes calculations of bootstrap current and fusion power based on prescribed density, temperature, and current (q) profiles.

The basic parameters used were based on Lang Lao's equilibrium 99825.00276:

$$
\begin{array}{ll}
\mathrm{a}=1.376 \mathrm{~m} & \mathrm{~B}_{\mathrm{T} 0}=7.982 \mathrm{~T} \\
\mathrm{R}=5.524 \mathrm{~m} & \mathrm{I}=8.267 \mathrm{MA} \\
\kappa=1.714 & \mathrm{q}(0)=6.105 \\
\delta=0.631 &
\end{array}
$$

Other parameters assumed are:

$$
\begin{array}{lll}
\left\langle\mathrm{n}_{\mathrm{e}}\right\rangle=2.085 \times 10^{20} \mathrm{~m}^{-3} & \left.\mathrm{n}_{\mathrm{e} 0} / \mathrm{n}_{\mathrm{e}}\right\rangle=1.27 & \mathrm{~N}_{\text {Greenwald }} \leq 1.5 \\
\left\langle\mathrm{~T}_{\mathrm{e}}\right\rangle=10.09 \mathrm{keV} & \mathrm{T}_{\mathrm{e} 0} /\left\langle\mathrm{T}_{\mathrm{e}}\right\rangle=2.12 & \\
\mathrm{n}_{\mathrm{He}} / \mathrm{n}_{\mathrm{e}}=0 & \mathrm{n}_{\mathrm{Fe}} / \mathrm{n}_{\mathrm{e}}=6.56 \times 10^{-4} & \mathrm{Z}_{\mathrm{eff}}=1.5 \\
\tau_{\mathrm{Ei}} / \tau_{\mathrm{Ee}}=5 & \varepsilon \beta_{\mathrm{p}} \leq 1\left(\beta_{\mathrm{p}} \leq 4.0\right) & \beta_{\mathrm{N}} \leq 5.1
\end{array}
$$

This gives:

$$
\begin{array}{lll}
\beta_{\mathrm{N}}=5.06 & \beta_{\mathrm{p}}=3.31 & \beta=3.81 \% \\
\mathrm{~W}=500 \mathrm{MJ} & & \\
\mathrm{P}_{\alpha}=588 \mathrm{MW} & \mathrm{H}_{98 \mathrm{y} 1}=1.12 & \mathrm{H}_{89 \mathrm{p}}=2.37
\end{array}
$$

Because the bootstrap calculation does not include geometric effects such as the Shafranov shift, the calculated bootstrap current is multiplied by 0.73 in order to match the reference equilibrium. This leads to an underestimate of the bootstrap current at low beta.

The parametrization of the $q$ profile yields $q_{\min }=3.57$ at $\rho=0.52$, not quite matching the equilibrium, and also leading to a bit higher bootstrap current.

The external heat source is assumed to be a tangential $250 \mathrm{keV} \mathrm{D}^{\circ}$ beam, with a maximum power of $150 \mathrm{MW}$. The current drive associated with this beam is included in the calculation. 
The pressure profile is constructed to match the reference equilibrium. The electron density and temperature profiles (versus $\rho$ ) are:
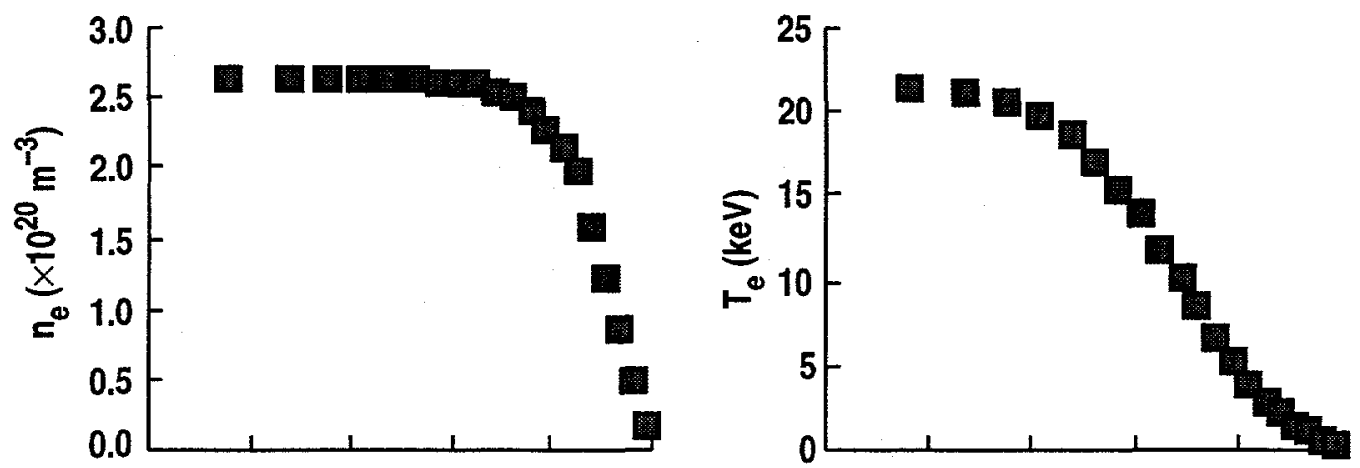

\subsection{RAMPUP SCENARIOS}

Three rampup scenarios were developed:

\subsubsection{Case A}

A constant confinement multiplier $\left(\mathrm{H}_{98 \mathrm{y}} \mathrm{l}=1.12\right)$ is maintained and the density is kept at $1.5 \mathrm{n}_{\mathrm{G}}$. We start at maximum $\beta_{\mathrm{p}}(=4.02)$, and increase the power and current until the maximum $P_{N B}(=150 \mathrm{MW})$ is reached. Then heating is continued at this power, while $\beta_{p}$ falls and $\beta_{N}$ rises. When $\beta_{N}$ reaches the limit (=5.1), the power is reduced, and the plasma is allowed to reach the final current and pressure.

The three phases are clearly seen in the I versus $t$ plot. The minimum initial current is about 1.1 MA.

\subsubsection{Case B}

We assume that confinement is externally controllable, up to a maximum of $\mathrm{H}_{98 \mathrm{y}} 1=$ 2 , and the density is maintained at $1.5 \mathrm{n}_{\mathrm{G}}$. Starting at maximum $\beta_{\mathrm{p}}$, with $\mathrm{H}_{98 \mathrm{y} 1}=2$, the beam power is adjusted as the pressure rises (the maximum needed is $30 \mathrm{MW}$ ). When no external power is needed, we gradually reduce the confinement multiplier. When the $\beta_{\mathrm{N}}$ limit is reached, we continue at constant $\beta_{\mathrm{N}}$ (reducing $\beta_{\mathrm{p}}$ ) until the operating conditions are reached.

For Case B, the minimum initial current is about $1.9 \mathrm{MA}$, and the ramp rate is about $1 / 3-1 / 4$ of Case $A$. 

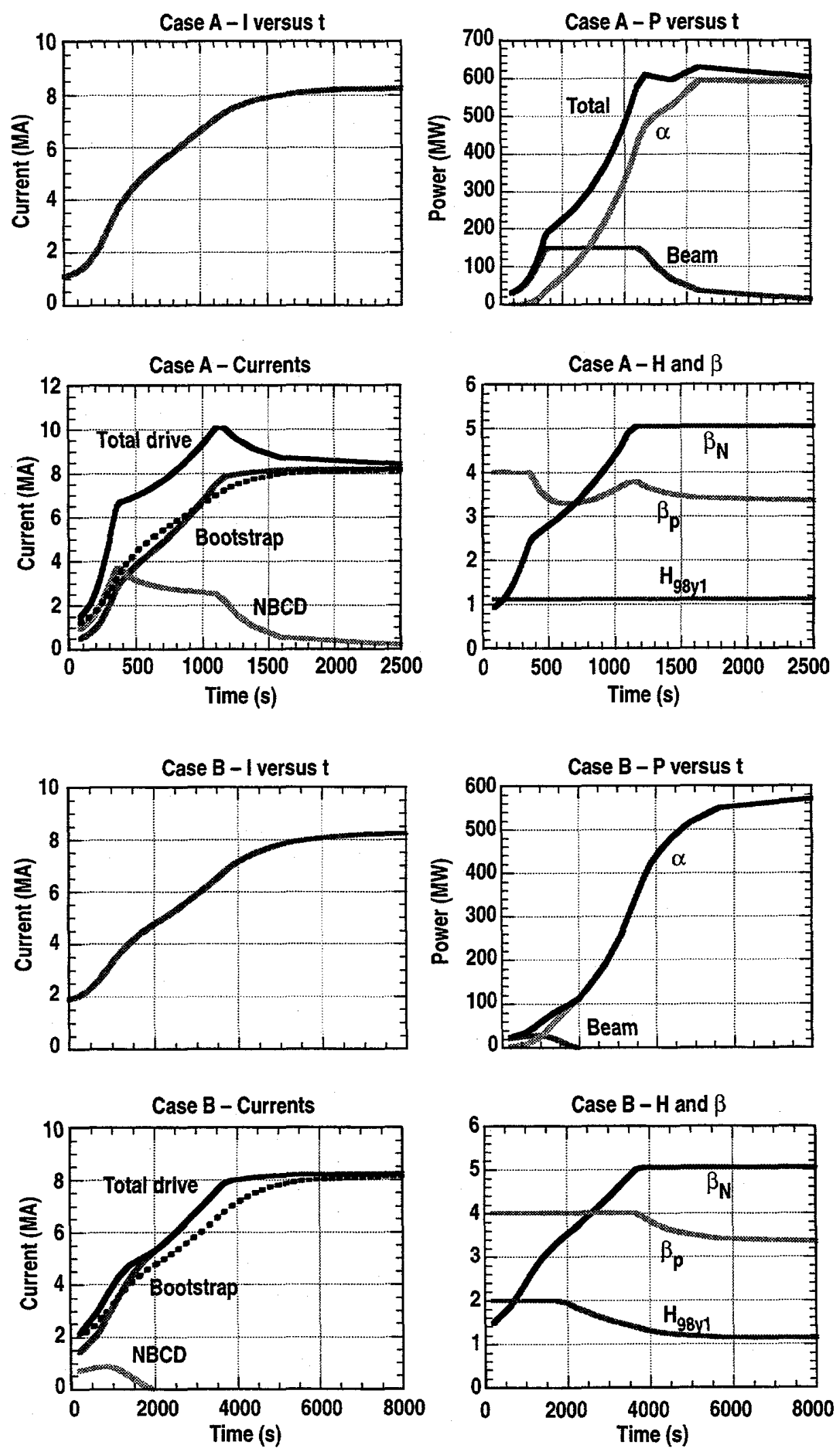


\subsubsection{Case C}

We maintain the density at $0.5 \mathrm{n}_{\mathrm{G}}$ up to $7 \mathrm{MA}$, then ramp it to $1.5 \mathrm{n}_{\mathrm{G}}$. The other rules are the same as case B. The maximum power needed is $130 \mathrm{MW}$. Note that one may choose any case intermediate between B and C, i.e., starting at low density to speed the rampup and increasing the density at some intermediate time.

The minimum initial current in Case $\mathrm{C}$ is about $0.8 \mathrm{MA}$. The current rises rapidly as long as the density is low. Starting the density increase at 7 MA leads to a rapid drop in beam power as the fusion power rises. The current ramp is drastically slowed because a large NBCD overdrive is replaced by a small bootstrap overdrive. Further optimization of the transition from low to high density would help.
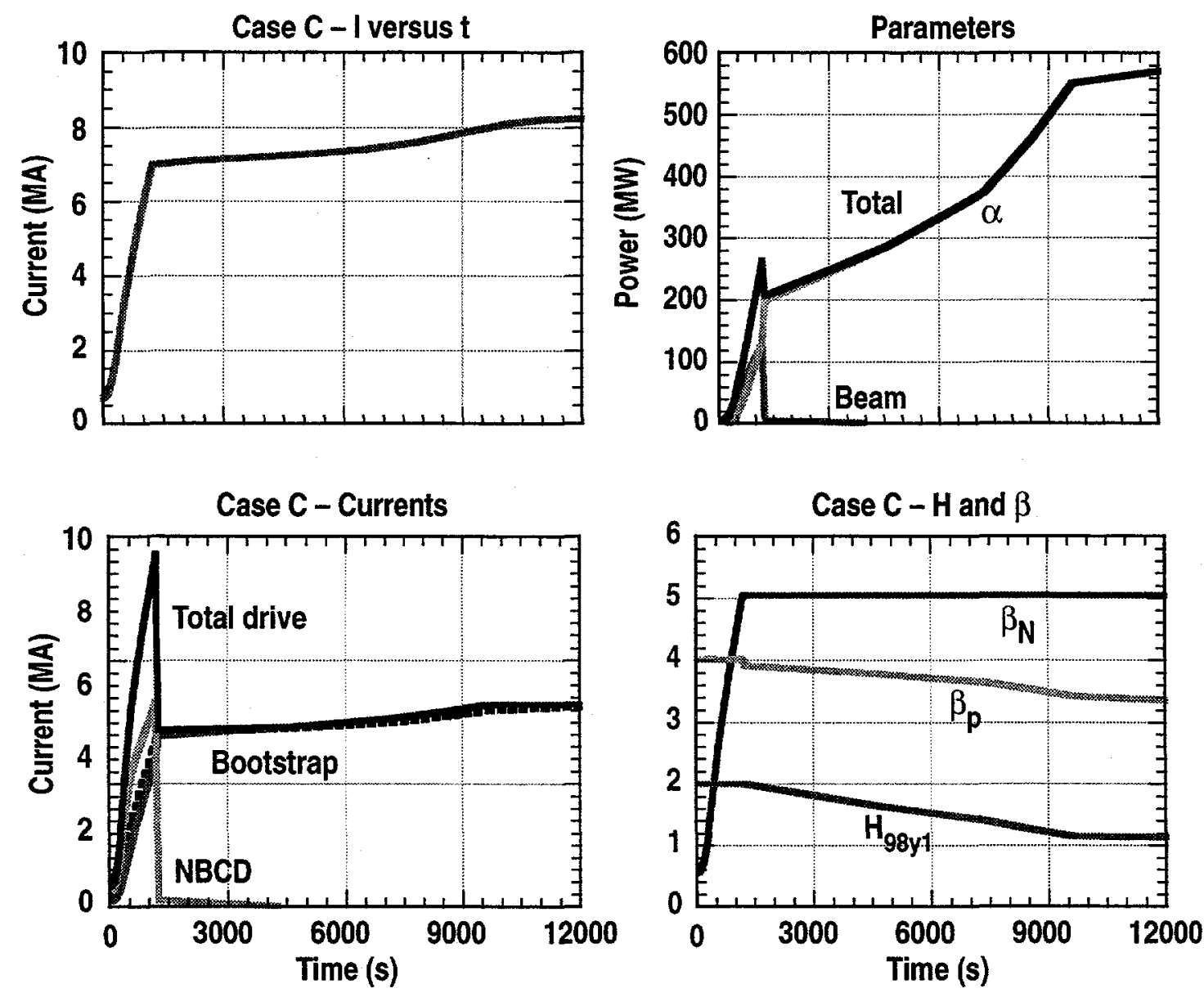


\section{SUMMARY AND DISCUSSION}

In the 1999 study, we have reaffirmed some previous conclusions and discovered new insights to key issues that are expected to have impact on the ARIES-AT design. To recapitulate the findings,

In stability optimization

- Ideal low $\mathrm{n}$ modes are stabilized by a conducting wall at $\sim 1.2 \mathrm{a}$

- Beta is limited by high $\mathrm{n}$ ideal ballooning modes near the plasma outer region

- Rotational drive and radially localized off-axis current drive are essential for stabilization against resistive wall modes and neo-classical tearing modes

In transport and current drive modeling

- The initial 13.2 MA, $\beta_{\mathrm{N}}=5.6$ design produces too much alpha heating

- Physics-based modeling with ITB indicates a smaller device should be considered

In divertor heat exhaust

- High radiated fraction of the total exhausted power $(>0.5)$ is essential to keep the peaked inboard and outboard heat fluxes at a manageable level $\left(<10 \mathrm{MW} / \mathrm{m}^{2}\right)$

- It is essential to accurately maintain the double-null magnetic balance (to $\sim 0.5 \mathrm{~cm}$ )

Based on these findings, we recommend the following future work:

- Explore dependence of pressure and bootstrap profiles on density profile and rotation profile

- Look for self-consistent pressure, bootstrap, density, and rotation profiles giving near optimal stability results

- Calculate rotation needed for stabilization of resistive wall mode and the resulting neutral beam requirements

- Perform power-balance

- Calculate UEDGE 2D solution for edge profiles

- Determine stabilization requirements for neoclassical tearing modes 


\section{APPENDIX A}

In this appendix, we include a brief look at helicity injection for transformerless operation. This work was originally done for the ARIES-ST study in 1998 but is expected to have relevance for the ARIES-AT as well.

\section{ST STARTUP: HELICITY INJECTION}

Spherical tokamak reactor plasmas must be formed without a central induction coil. If an initial "target" plasma having $\mathrm{I}_{\mathrm{p}} \geq 0.35 \mathrm{MA}$ can be generated by some means, then the plasma current can be ramped up to its full operating value by a combination of bootstrap and conventional external non-inductive current drive. This section presents the requirements for target plasma formation by helicity injection (HI). A new helicity injection configuration is proposed that overcomes many of the design and engineering difficulties posed by the divertor electrodes and insulators required by conventional $\mathrm{HI}$.

Non-inductive helicity injection consists of driving electric current via magnetized electrodes. The current flows mainly parallel with the magnetic field, which connects the source electrodes to the plasma volume. The concept of helicity enters for two reasons. First, magnetic helicity is a quantitative measure of the linkage of poloidal and toroidal magnetic fluxes, and in an axisymmetric toroidal system the poloidal flux is intimately related to toroidal plasma current. Second, magnetic helicity is not only a globally conserved quantity in ideal MHD, but it is also nearly conserved in resistive plasmas, even through violent reconnections and relaxations. Therefore, helicity conservation simplifies current injection calculations under conditions where helicity is approximately conserved. When a plasma is started by HI alone, the current along the magnetic field must exceed a critical value at which the magnetic lines and current bend and bulge into the vessel. A turbulent helicity-conserving relaxation follows, in which the plasma reaches an equilibrium with vessel image currents and any externally applied shaping and equilibrium poloidal magnetic field. It does not matter that the formation process is turbulent, as long as the plasma becomes quiescent when the HI current is turned off.

Helicity preserving current injection from magnetized electrodes has been demonstrated in spheromaks [1,2] and in STs, especially in the Helicity Injected Tokamak (HIT) [3], where $\mathrm{I}_{\mathrm{p}}=0.25 \mathrm{MA}$ has been produced. This plasma current is already $70 \%$ of the ARIES-ST minimum target plasma requirement. In HIT the electrode 
current is about $0.15 \mathrm{I}_{\mathrm{p}}$, or about $50 \mathrm{kA}$ for $\mathrm{I}_{\mathrm{p}}=0.35 \mathrm{MA}$. It is not known whether this proportion will be the same in ARIES-ST.

Helicity injection in HIT and in NSTX (an ST just beginning operation) uses the split vessel topology illustrated in Fig. 1(a), in which the inner and outer portions of the vacuum vessel serve as the two electrodes. The two parts of the vessel must be electrically insulated from one another. The electrode magnetic flux is supplied by the divertor coil(s). A short pulse of fundamental resonance $\mathrm{ECH}$ can be used to assist the initial gas breakdown and thereby reduce the peak voltage requirement across the vessel gap. The NSTX experiments will test the scale up of this $\mathrm{HI}$ configuration to $\mathrm{I}_{\mathrm{p}} \sim 1 \mathrm{MA}$ and $a \sim 0.65 \mathrm{~m}$. Figure 1(b) illustrates an alternate topology in which the divertor "dome" doubles as an electrode. The divertor dome electrode must then be insulated from the rest of the vessel, but the vessel itself need not be split and insulated (a divertor dome is sometimes used to promote high divertor particle recycling and radiation).

Even though the configurations (a) and (b) can make the target plasma, they are unsatisfactory for a fusion reactor, because the electrical insulators are rapidly destroyed by $14 \mathrm{MeV}$ neutron bombardment. Furthermore, large insulators are difficult to cool, especially in the divertor, which has the largest steady heat fluxes in the reactor. Also, sputtering of divertor target material coats the insulators with conducting material in short order.

Another potential problem stems from the need to puff a large amount of gas, if the cathode electrode is not a strong electron emitter. This gas enters the plasma and might raise its density too high. A non-emitting cathode operates by repelling electrons and

(a)

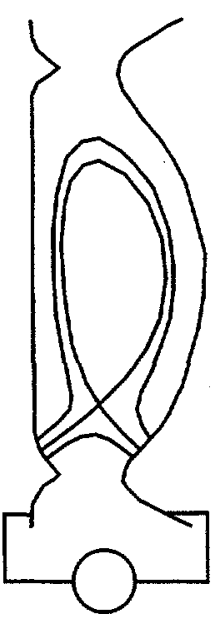

(b)

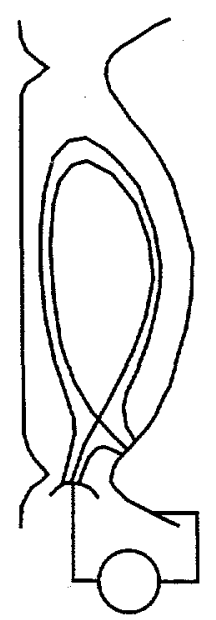

(c)

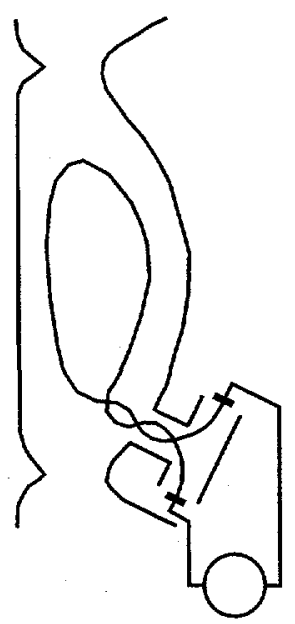

Fig. A-I. Three helicity injection concepts. (a) Split vessel. (b) Divertor dome doubling as electrode.(c) External electrodes. 
collecting positive ions from the nearby plasma, and the cathode must be supplied with sufficient gas to support the ion current. If the geometry around the cathode does not induce a high degree of local gas recycling and reionization, then as much as one hydrogenic ion must be injected for each electronic charge driven between the electrodes. This amounts to about $3 \times 10^{23}$ atom/s for a $50 \mathrm{kA}$ electrode current, which would give an average target plasma density (plasma volume $\sim 1000 \mathrm{~m}^{3}$ and gas puff limited to $10 \mathrm{~ms}$ ) of $3 \times 10^{18} \mathrm{~m}^{-3}$. This density is also the Greenwald limiting density at $\mathrm{I}_{\mathrm{p}}=0.35 \mathrm{MA}$. Any higher density would be problematical. Therefore, unless the gas is handled carefully, the target plasma density with internal non-emitting electrodes will be too high.

In view of all the problems with electrodes inside the vessel, a new external electrode helicity injection concept, illustrated in Fig. 1(c), is proposed. Here the initial plasma and current is generated by a source outside of the blanket and injected as a freely propagating, relaxed Taylor double helix plasma [4] through a port at any convenient location. The plasma is struck in the cross tube between two magnetized electrodes, and the electrode current is increased rapidly to where the linear plasma pinch column kinks into the injection tube, which must have at least twice the area of the pinch tube. The plasma twists itself into a Taylor double helix along the injection tube and then expands into the ST vessel. A similar injection technique generated spheromak plasmas successfully in the CTX experiment [5], but the technique has not been tested on an ST, nor has it been tested at an arbitrary location far from the divertor. The inner surface of the injection tube must be a good electrical conductor to guide the double helix plasma by image currents. The tube must by shielded from the ST toroidal magnetic field, which is already present at the time of startup. If $\mathrm{B}_{\mathrm{T}}$ is below $1 \mathrm{~T}$ at startup, the shielding can be accomplished by an iron insert, though the insert should be retracted after startup to avoid a permanent large magnetic error. Alternatively, the injection tube can be shielded by pulsing current through a shield coil, but the coil should be retracted so that its electrical insulation is not damaged later by neutrons.

Parameters of the double helix injection system can be estimated from helicity balance. Consider an ST target plasma with $R_{0}=3.2 \mathrm{~m}, \mathrm{a}=2.0 \mathrm{~m}, \kappa=\mathrm{b} / \mathrm{a}=3.785$ and $\mathrm{B}_{\mathrm{T}}=1.0 \mathrm{~T}$ at startup. Let the desired target plasma current be $\mathrm{I}_{\mathrm{p}}=0.5 \mathrm{MA}$, larger than the 0.35 MA minimum specification. This plasma has a magnetic helicity content of $\mathrm{K}=2 \int \psi_{\text {tor }} \mathrm{d} \psi_{\text {pol }} \approx \psi_{\text {tor }} \psi_{\text {pol }} \approx(48 \mathrm{wb})(0.5 \mathrm{wb})=24 \mathrm{wb}^{2}$, where $\psi_{\text {tor }}$ and $\psi_{\text {pol }}$ are the plasma toroidal and poloidal magnetic fluxes, respectively. Helicity propagates along the double helix at the rate $\mathrm{K}_{\mathrm{DH}}^{\prime}=2 \psi_{\mathrm{DH}} \mathrm{U}_{\mathrm{DH}} \mathrm{wb}^{2} / \mathrm{s}$, where $\psi_{\mathrm{DH}}$ is the longitudinal magnetic flux along one half (outward or return) of the double helix, and $U_{D H}$ is the electric potential difference between the two halves. In order to build up the target plasma 
in, say, $0.1 \mathrm{~s}$, helicity balance requires helicity injection at the rate $\mathrm{K}_{\mathrm{DH}}^{\prime}=\mathrm{K} /(0.1 \mathrm{~s})=$ $240 \mathrm{wb}^{2} / \mathrm{s}$. If we choose $\mathrm{a}_{\mathrm{DH}}=0.3 \mathrm{~m}$ for the injection tube and $\mathrm{B}_{\mathrm{DH}}=\mathrm{B}_{\mathrm{T}}=1 \mathrm{~T}$, then $\psi_{\mathrm{DH}}=0.14 \mathrm{wb}^{2}$ and $\mathrm{U}_{\mathrm{DH}}$ must be at least $860 \mathrm{~V}$. The voltage difference between the source electrodes will have to be somewhat larger, to accommodate inefficiencies. However, it should be no more than a few $\mathrm{kV}$, which is a technically reasonable value. The longitudinal current in one half of the double helix, $\mathrm{I}_{\mathrm{DH}}$, is set by the eigenvalue for its existence in a tube of radius $a_{D H}: I_{D H} / \psi_{D H}=3.11 / \mu_{0} a_{D H}$. This computes to $I_{D H} \approx$ 1.2 MA, which is larger than the ST toroidal current. This current inefficiency is a well known consequence of injecting helicity through a small port whose radius is much less than the radius of the toroidal plasma. The electrode current will be larger still, perhaps 1.5 or $2 \mathrm{MA}$.

In summary, the ARIES-ST target plasma current is less than twice what has already been achieved by helicity injection in the small HIT experiment. The usual HI configurations, which require electrical insulation and high gas puffing within the vessel, are incompatible with a fusion reactor reactor. A new HI configuration with external electrodes and double helix plasma injection through an injection tube is proposed. Although it is inefficient, it avoids the problems of conventional helicity injection concepts.

\section{REFERENCES}

[1] T.R. Jarboe et al., Phys. Rev. Lett. 51 (1983) 39.

[2] P.K. Browning et al., Phys. Rev. Lett. 68 (1992) 1718.

[3] B.A. Nelson et al., Phys. Rev. Lett. 72 (1994) 3666.

[4] J.B. Taylor, Proc. 5th IAEA Conference on Plasma Physics and Controlled Fusion (Tokyo, 1974) vol. 1 (1975) 161.

[5] J.C. Fernandez et al., Phys. Fluids B 1 (1989) 1254. 